\title{
Physical and Predictive Models of Ultrathin Oxide Reliability in CMOS Devices and Circuits
}

\author{
James H. Stathis
}

\author{
Invited Paper
}

\begin{abstract}
The microelectronics industry owes its considerable success largely to the existence of the thermal oxide of silicon. However, recently there is concern that the reliability of ultra-thin dielectrics will limit further scaling to slightly thinner than $\mathbf{2} \mathbf{~ n m}$.

This paper will review the physics and statistics of dielectric wearout and breakdown in ultrathin $\mathrm{SiO}_{2}$-based gate dielectrics. Electrons or holes tunneling through the gate oxide generate defects until a critical density is reached and the oxide breaks down. The critical defect density is explained by the formation of a percolation path of defects across the oxide. Only $<1 \%$ of these paths ultimately lead to destructive breakdown, and the microscopic nature of these defects is not known. The rate of defect generation decreases approximately exponentially with supply voltage, below a threshold voltage of about $5 \mathrm{~V}$ for hot electron-induced hydrogen release. However, the tunnel current also increases exponentially with decreasing oxide thickness, leading to a decreasing time-to-breakdown and a diminishing margin for reliability as device dimensions are scaled. Estimating the reliability of the dielectric requires an extrapolation from the measurement conditions (e.g., higher voltage) to operation conditions. Because of the diminished reliability margin, it has become imperative to try to reduce the error in this extrapolation. Long-term $(>1$ year) stress experiments are now being used to measure the wearout and breakdown of ultrathin $(<2 \mathbf{n m})$ dielectric films as close as possible to operating conditions. These measurements have revealed the details of the voltage dependence of the defect generation rate and critical defect density, allowing better modeling of the voltage dependence of the time-to-breakdown. Such measurements are used to guide the technology development prior to the manufacturing stage. We then discuss the nature of the electrical conduction through a breakdown spot and the effect of the oxide breakdown on device and circuit performance. In some cases, an oxide breakdown does not lead to immediate circuit failure, so more research is needed in order to develop a quantitative methodology for predicting the reliability of circuits.
\end{abstract}

Index Terms-Dielectric breakdown, MOSFETs, reliability.

\section{INTRODUCTION}

$\mathbf{T}$ HE microelectronics industry, including the Internet and the telecommunications revolutions, owes its success largely to the existence of the thermal oxide of silicon, i.e., silicon dioxide $\left(\mathrm{SiO}_{2}\right)$. A thin layer of $\mathrm{SiO}_{2}$ forms the insulating layer between the control "gate" and the conducting "channel"

Manuscript received February 7, 2001

The author is with the IBM Research Division, T. J. Watson Research Center, Yorktown Heights, NY 10598 USA (e-mail: stathis@us.ibm.com).

Publisher Item Identifier S 1530-4388(01)04252-4.

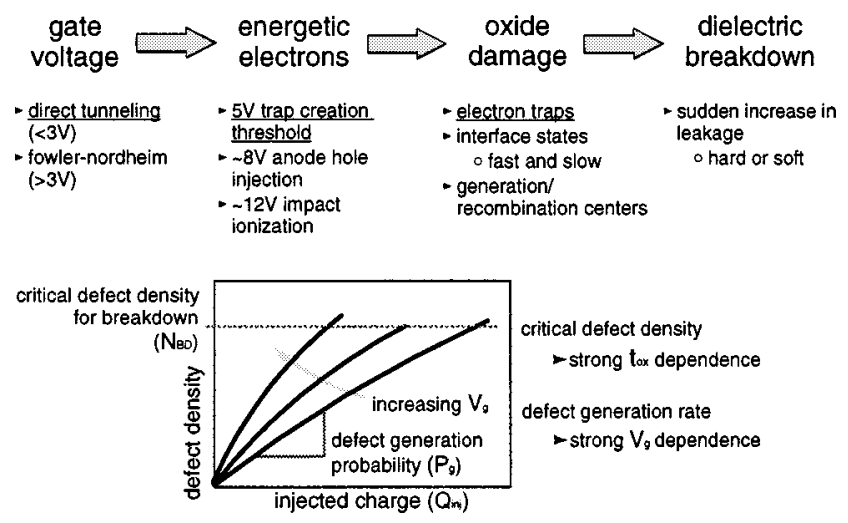

Fig. 1. Mechanism of defect generation to breakdown.

of the transistors used in modern integrated circuits. As circuits are made more dense, all the dimensions of the transistors are reduced ("scaled") correspondingly [1], so that nowadays the $\mathrm{SiO}_{2}$ layer thickness $\left(t_{\mathrm{ox}}\right)$ is $2 \mathrm{~nm}$ or less, and the reliability of such ultrathin oxide layers has become a major concern for continued scaling.

The reliability of $\mathrm{SiO}_{2}$, i.e., the ability of a thin film of this material to retain its insulating properties while subjected to high electric fields for many years, has always been an important issue and has been the subject of numerous publications over the last 35 years [2]-[6], since the realization that $\mathrm{SiO}_{2}$ could be used as an insulating and passivating layer in silicon-based transistors [7], [8]. Oxide reliability and the experimental methods for accelerated testing have been the subject of earlier review papers [9]-[18].

For the relatively thick $(>10 \mathrm{~nm})$ oxides used in earlier technologies, the breakdown mechanisms are actually fairly complex, and the detailed understanding of the intrinsic reliability has only come about in the more recent past, as manufacturing processes have matured and high-quality MOSFET samples incorporating a wide range of oxide thickness have become available for scientific study [19]. The essential elements of our present understanding are illustrated in Fig. 1. When a voltage is applied across the gate oxide, an electron current will flow if the gate voltage $\left(V_{g}\right)$ is high enough and/or the oxide is thin enough. For thick oxides, the current is controlled by Fowler-Nordheim tunneling [20], [21], while for thin oxides $\left(t_{\mathrm{ox}} \lesssim 3 \mathrm{~nm}\right.$ ) at voltages below about $3 \mathrm{~V}$ (corresponding to the barrier height between n-type silicon and the $\mathrm{SiO}_{2}$ ) the current is due to direct quantum-mechanical tunneling. The 
electrons flowing across the oxide will trigger several processes depending on their energy. At least three defect generation mechanisms have been identified: impact ionization and anode hole injection occur at higher voltages, leading to hole trapping and hole-related defect generation [19], [22]. The lowest energy process so far identified, which dominates at the voltages where present MOSFETs operate, is the so-called "trap creation" process attributed to hydrogen release from the anode [23] with a threshold gate voltage of about $5 \mathrm{~V}$. This process continues in the subthreshold region even at operating voltages, down to $1.2 \mathrm{~V}$ or lower [24], [25].

As a consequence of the reaction of the released mobile hydrogen [23], [26]-[30], a variety of defects such as electron traps, interface states, generation/recombination centers in the substrate, positively charged donor-like states, etc., gradually build up in the oxide. For breakdown, it is believed that bulk traps are the most important [31]-[34]. Eventually, enough damage builds up to the point where the oxide breaks down destructively. Breakdown is defined experimentally as a sudden increase in conductance, often accompanied by current noise.

\section{Physical Models for Defect Generation}

The evidence for hydrogen involvement in defect generation and breakdown is circumstantial but strong. Already in the 1970s it was suggested that hydrogen would play a major role in the growth of $\mathrm{SiO}_{2}$ and in radiation processes in this material [35], [36]. The evidence up to 1989 for hydrogen's role in the trap creation process has been reviewed by DiMaria [23]. Since that time, it was shown that exposure of bare $\mathrm{SiO}_{2}$ films to atomic hydrogen radicals, in the absence of any electric field, will produce electrically active defects essentially identical to those produced by electrical stress or radiation [26], [37]-[51]. Paramagnetic interface defects [38], [39], [48], [50] (the $P_{b}$ centers, which are $\mathrm{Si}$ dangling bonds at the $\mathrm{Si} / \mathrm{SiO}_{2}$ interface), diamagnetic interface defects (fast and slow interface states) [37], [38], [40]-[43], [45]-[50], and bulk electron traps [44] are produced. The desorption rate of hydrogen from Si surfaces was measured as a function of incident electron energy [52] and showed a dependence remarkably similar to the voltage dependence of the trap generation process [23], [25]. However, a major perceived stumbling block to the general acceptance of the "hydrogen model" for breakdown has been the apparent lack of any isotope effect for the breakdown process [53] compared to the large effect observed for hydrogen/deuterium desorption and for channel hot electron-induced interface degradation [54]. This may have recently been resolved by the observation [55], [56] of a significant isotope effect on the stress-induced flat-band voltage shift and stress-induced leakage current (SILC), which is a measure [44], [57], [58] of the bulk traps which ultimately relate to breakdown. A significant isotope effect on trap generation and oxide breakdown in deuterated oxide has also been reported earlier [59].

Two other physical models for breakdown have been widely discussed in the literature. The first is the anode-hole injection (AHI) model, which claims that breakdown is caused by holes which are injected from the anode contact [60], [61]. This model derived considerable support from a comprehensive theoretical treatment of anode hole injection by surface plasmon

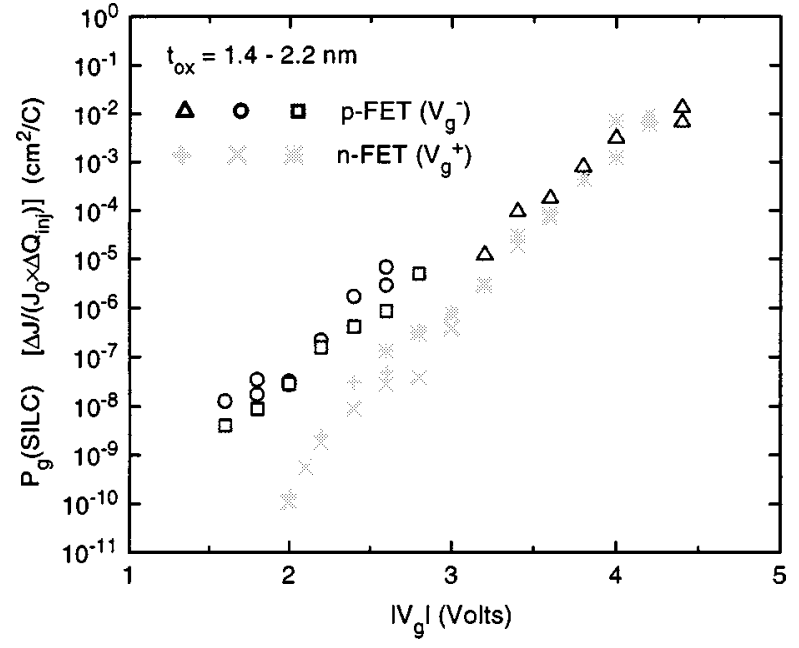

Fig. 2. Comparison of the defect generation probability $\left(P_{g}\right)$ for holes (p-FET) or electrons (n-FET)

excitations, decaying into electron-hole pairs in the silicon gate [62], [63], and experimental data showing the expected dependence on anode material [62], [64]. The original concept was a variant of earlier models [3], [4], [6] postulating a positive feedback mechanism for current runaway, caused by the field-enhancement due to the trapped holes. The main experimental evidence in support of this was a constant value of the hole fluence to breakdown as a function of oxide field [60] $Q_{p} \approx$ $0.1 \mathrm{C} / \mathrm{cm}^{2}$, where the injected hole flux is obtained from the substrate hole current using n-FETs biased in inversion, although it was later shown that $Q_{p}$ decreases for $t_{\mathrm{ox}}$ less than about $6 \mathrm{~nm}$ [61] and that the constancy of $Q_{p}$ does not hold at temperatures below $300 \mathrm{~K}$ [65]. The AHI model is commonly associated with a time-to-breakdown $\left(T_{\mathrm{BD}}\right)$ dependence on oxide field of the form $T_{\mathrm{BD}} \propto \exp \left(-\right.$ constant $\left./ E_{\mathrm{ox}}\right)$ (referred to as the " $1 / E$ model") [66], but since this dependence comes mostly from the form of the Fowler-Nordheim current it is not an essential element of the physical model and would not be expected to hold in the direct tunneling regime [67].

According to the plasmon model calculations [62], the gate voltage threshold for positive charge generation by hole trapping due to AHI is 7-8 V for FETs with $\mathrm{n}^{+}$-poly gates, and this was confirmed experimentally [22]. Thus, this mechanism may not be able to account for the substrate currents measured at lower voltage. A recent modification of the AHI model [68], [69] proposes that a weaker minority carrier ionization process [70] is responsible for hole injection and defect generation at low voltages. This mechanism will be operative for electron injection into a p-type material or hole inversion layer, and was observed for n-FETs with low gate doping when the n-poly gate inverted [34]. The modified model can successfully fit the measured slope of $T_{\mathrm{BD}}$-versus-voltage at high fields [67], [69] but cannot account for the absolute magnitude of the defect generation rate. Since the hole current at low voltage (e.g., 2-3 V) in this model is at least 12 orders of magnitude lower than the primary electron current [68], the defect generation rate per hole must be very much greater than the rate per injected electron. However, direct measurement of the rate of defect generation by holes transporting through an $\mathrm{SiO}_{2}$ layer [71], [72] shown in Fig. 2 gives values comparable to the generation rate due to 


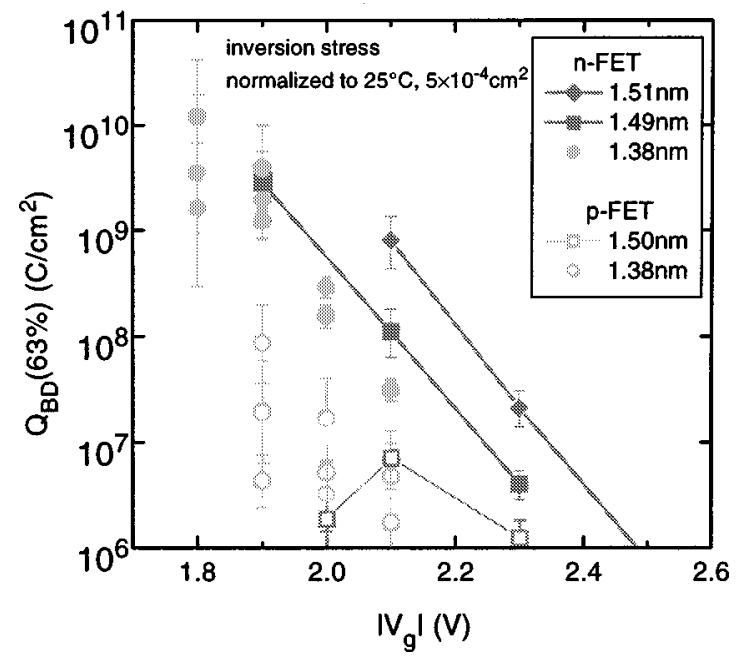

Fig. 3. Charge-to-breakdown measurements on p-FETs and n-FETs under inversion conditions.

electrons and many orders of magnitude less than what is required by a minority carrier mechanism. (See the next section for the definition of $P_{g}$.) Charge-to-breakdown measurements using substrate hot hole injection [73], [74] and on p-FETs at low bias [72] (Fig. 3) have also shown that the hole fluence to breakdown, $Q_{p}$, is as large as eight orders of magnitude greater than the value used in the AHI model, consistent with the measured defect generation rate. The data in Fig. 3 indicate that p-FET breakdown may be the limiting factor at low voltage, contrary to the usual idea [75] that n-FETs should represent the worst case. The AHI model has also been criticized on the basis that the measured substrate currents at low voltage may have other origins besides hole tunneling through the oxide, including generation-recombination processes in the substrate [22] and photoexcitation due to photons generated by hot electrons in the gate [22], [76]. Other kinds of defect generation, especially donor-like interface states which give interfacial positive charge, or bulk neutral traps which cause an increase in the leakage current, can be easily mistaken for trapped holes in the oxide [19], [22], [44]. In cases where holes due to AHI are clearly observed (i.e., above 7-8 V) [22] there is no correlation with breakdown [19], [77].

The other widely cited breakdown model is the "thermochemical" model, or " $E$-model," which proposes that defect generation is a field-driven process and that the current flowing through the oxide plays at most a secondary role. The development of this model from its origin in the mid 1980s through the late 1990s has been reviewed by McPherson [78], [79]. Briefly, the model considers the interaction of the applied electric field $E$ with the dipole moments associated with oxygen vacancies (weak $\mathrm{Si}-\mathrm{Si}$ bonds) in $\mathrm{SiO}_{2}$. The activation energy required for bond breakage is lowered by the dipolar energy, leading to a quantitative prediction for the field dependence of the activation energy for dielectric breakdown (or equivalently, the temperature dependence of the field acceleration factor) which agreed well with experiment [80]-[82]. McPherson also showed [78] that allowing for a distribution of energies of the weak bonds could account for a wide range of observations of the temperature- and field-dependence of $\mathrm{SiO}_{2}$ breakdown times, since the

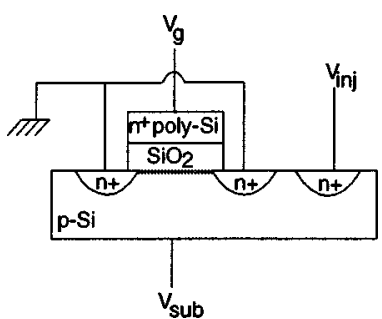

Fig. 4. Device structure for SHE injection.

defect which dominates the breakdown process may change depending on stress conditions. However, it was also observed that for very thin oxides ( $t_{\mathrm{ox}} \lesssim 4 \mathrm{~nm}$ ) the breakdown times are no longer a function of only the field, but are strongly decreasing with thickness at the same oxide field [79], [83]. The decreasing breakdown times are consistent with the increasing direct-tunneling leakage currents in the ultrathin oxides. It was proposed that the strong increase in current leads to an increase in hole injection (presumably by the same mechanism as in the AHI models discussed above), and that these holes are trapped at oxygen vacancies further reducing the activation energy for bond rupture [79], [83]. Other attempts to unify the AHI and thermochemical models by treating these as parallel competing mechanisms have been published [84]-[86].

The $E$-model has attained widespread acceptance, largely on the basis that, empirically, the $T_{\mathrm{BD}}$ data appear to follow an exponential dependence on field [80]-[82], [87]-[90] including an experiment of 3-yr duration at oxide fields down to 5.3 $\mathrm{MV} / \mathrm{cm}$ on $9-\mathrm{nm}$ films [91]. However, it must be pointed out that the exponential dependence on field is not proof of the validity of the particular physical model. Probably the strongest evidence against the thermochemical model comes from substrate-hot-electron (SHE) injection experiments [92], [93]. In this experiment, using a specially designed n-FET structure [94] (see Fig. 4), a forward-biased $n^{+}$diffusion in the p-type substrate is used to inject electrons toward the inversion layer at the $\mathrm{Si} / \mathrm{SiO}_{2}$ interface. The inversion layer is maintained by keeping the gate positively biased relative to the source and drain (both held at ground). A negative substrate bias accelerates the injected electrons, and the injector current can be controlled independently of both the substrate and gate bias by varying the magnitude of the forward bias applied across the injector junction. The substrate voltage controls the maximum electron energy incident on the $\mathrm{Si} / \mathrm{SiO}_{2}$ interface, and for a sufficiently high substrate bias so that some electrons are injected over the energy barrier into the oxide, the gate voltage additionally controls the energy of the electrons incident on the gate-oxide interface. In these experiments, it was found that the charge-to-breakdown $\left(Q_{\mathrm{BD}}\right)$ is strongly dependent on the substrate bias, even though the oxide field is held fixed [93]. Therefore, $Q_{\mathrm{BD}}$ correlates with the electron energy not the oxide field. This was also demonstrated for conventional Fowler-Nordheim stress by varying the doping of the anode [34], [95]. The SHE experiments also showed that $T_{\mathrm{BD}}$ is inversely related to the current density [93], again showing that breakdown is dominated by the effect of the energetic electrons and not the field in the oxide. The same conclusion was arrived 


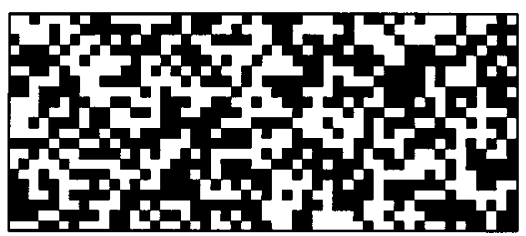

(a)

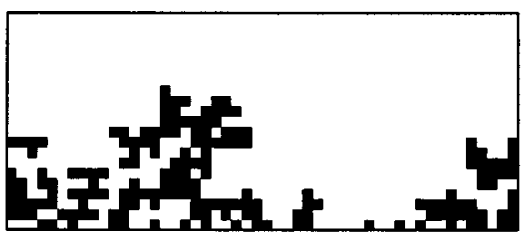

(b)

Fig. 5. Schematic of the percolation model. (a) Occupied sites $\left(p_{0}=0.5\right)$. Two-dimensional simple cubic lattice with uniform 50\% occupancy. "Defects" are represented by dark squares. (b) Cluster \#1, connects to bottom face. Starting from the same defect distribution as in (a), only those occupied sites which connect to the bottom face via nearest neighbors are kept. Note the use of periodic boundary conditions in the horizontal dimension. For this sample size and defect density, there is no breakdown path connecting the bottom face to the top face, but connecting paths would exist if the sample were made thinner or wider. This schematic shows a small $(21 \times 50)$ sample in two dimensions. The actual calculations were performed in three dimensions using a larger sample.

at based on measurements of the effect of varying the gate doping [34], [75], [96].

\section{The CRitical Defect Density}

The idea of damage building up to a critical level has been a key insight in leading to a predictive model of oxide reliability. The concept is not novel: for example, in 1983, resonant tunneling via defect states was proposed as a mechanism leading to breakdown, [97] and in 1986 it was proposed that defect generation ("wear out") above some threshold concentration would lead to a new conduction path resulting in oxide breakdown [98]. Nor does the concept depend in any way on the physics of defect generation, whether hydrogen-induced or otherwise: the field-driven model [78], [79], [83] ( $E$-model) also assumes a critical density of broken bonds in order to induce electric breakdown and thermal runaway, and the field dependence of trap generation has been used to support this model [99]. The most recent version of the AHI model [69], [100] likewise adopts the viewpoint that holes create some (unspecified) form of defect which eventually leads to a critical conduction path.

Beginning in the early 1990s, it was reported that in a large variety of oxide thickness stressed over a wide range of voltages, the charge-to-breakdown $\left(Q_{\mathrm{BD}}\right)$ is inversely related to the initial rate of defect generation for most stress conditions [19], [88], [101]-[103]. Extending this to thinner samples, the thickness dependence of oxide breakdown was explained as resulting from a thickness-dependent number of defects $\left(N^{\mathrm{BD}}\right)$ required to trigger breakdown [24]. The thickness dependence of $N^{\mathrm{BD}}$ was explained in terms of a simple percolation model [24], [31]-[33].

The relationship between the charge-to-breakdown $\left(Q_{\mathrm{BD}}\right)$, the critical defect density $\left(N^{\mathrm{BD}}\right)$, and the defect generation probability per injected electron density $\left(P_{g} \equiv q d N / d Q\right)$ is

$$
N^{\mathrm{BD}}=\frac{1}{q} \int_{0}^{Q_{\mathrm{BD}}} P_{g} d Q
$$

where $q$ is the magnitude of the electron charge. When the defect generation is first order in the electron fluence, then

$$
Q_{\mathrm{BD}}=q N^{\mathrm{BD}} / P_{g}
$$

(It is commonly observed that the defect density $N$ is a sublinear power-law function of the injected charge $Q$. However, observed over a sufficiently wide range of fluence, the full dependence is typically sigmoidal, with a linear region bracketed by sublinear portions at low and high fluence [72], [104]. If the low-fluence background is subtracted, a linear behavior is found independendent of stress conditions. At higher fluence (near breakdown) the saturation may be due to measurement technique, and the underlying defect generation probably continues in proportion to the fluence [105].)

The concept of a critical defect density was quantitatively examined by Suñé et al., [106] who showed that it leads to the correct statistical behavior. This was not a predictive model since $N^{\mathrm{BD}}$ was treated as a fitting parameter. Later, Degraeve [31] formulated the percolation model in which breakdown is envisioned as the formation of a connecting path of defects, as a result of random defect generation throughout the insulating film. This explains the thickness dependence of $N^{\mathrm{BD}}$ as a geometrical/statistical effect, by which a connecting path of defects is more likely for thinner films.

The percolation concept, and the origin of the thickness dependence of NBD, is schematically illustrated by the computer simulation [33] in Fig. 5. According to this model, breakdown can occur only when a connecting path of traps is formed across the gate oxide, forming a conducting path from the substrate to the gate. The probability of forming such a connecting path ("percolation path") with randomly generated defects throughout the oxide bulk is computed as a function of defect density and oxide thickness. For a given defect density, the formation of a percolation path is more likely for thinner oxides. Conversely, as the oxide is made thinner a percolation path can form with some probability at a lower average defect density than is necessary in a thick oxide. In the simulation, we start by placing defects (black squares) randomly throughout the sample [Fig. 5(a)], and then discard all defects which are not part of a cluster that is attached (via nearest neighbors) to one face of sample [Fig. 5(b)]. It does not matter at which face we begin when the percolation cluster is found, because if a site is part of a cluster which connects the two faces of the sample, then it will be counted regardless of which side we start from. It would be computationally redundant to separately examine the opposite cluster extending from other face of the sample, because this cluster cannot touch the first face unless the first cluster also spans the sample. (Note the use of periodic boundary conditions in the lateral direction, i.e., edge effects are avoided by treating the sample as if it were a cylinder made by connecting the left and right ends.) For the particular 


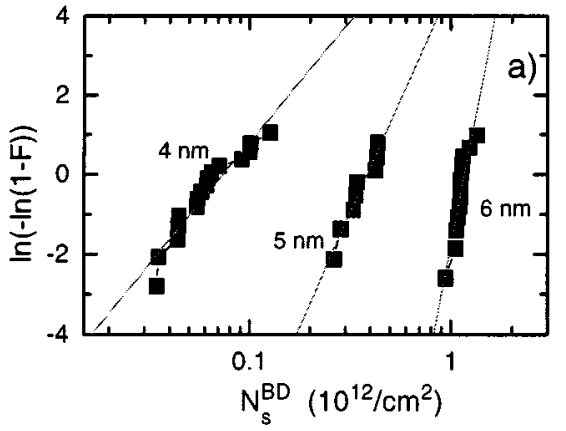

(a)

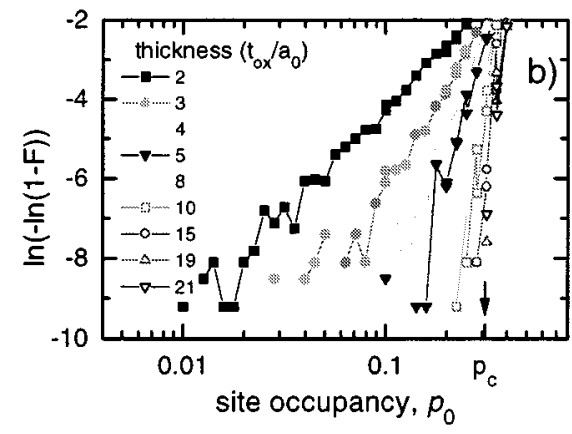

(b)

Fig. 6. (a) Weibull plot of the cumulative failure (F) distributions of measured defect density at breakdown, for p-FETs with oxide thickness from 4 to $6 \mathrm{~nm}$ under positive gate bias Fowler-Nordheim stress. The lines are first order least squares fits. (b) Weibull plot of the calculated cumulative failure (F) distributions for percolation on a 3-D simple cubic lattice as a function of site occupation fraction $p_{0}$, with normalized thickness as a parameter. The Weibull function is normalized to a unit area $a_{0}^{2}$, the defect cross-sectional area. The classical percolation threshold is indicated by $p_{c}$.

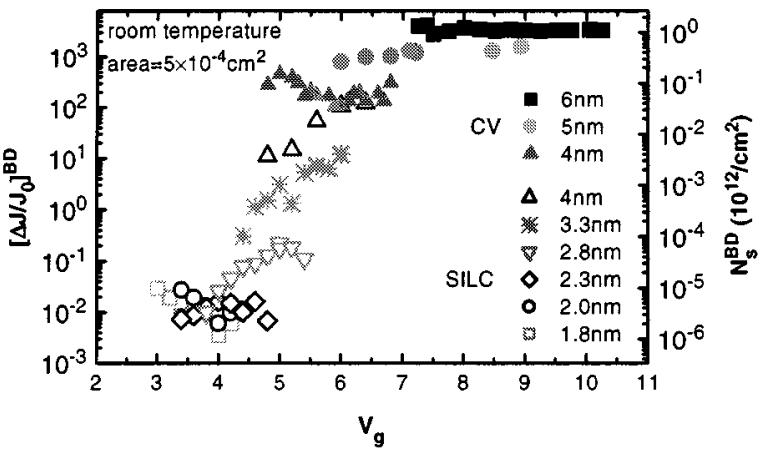

Fig. 7. Critical defect density at breakdown. Filled symbols from CV, open symbols from SILC. The CV and SILC data are normalized to their average values at $5 \mathrm{~nm}$.

defect density illustrated in Fig. 5, there is no percolation path connecting top to bottom of the sample, and therefore we have not reached breakdown. However, breakdown would have occurred at this density if the sample were thinner, i.e., thinner than the extent of the cluster in Fig. 5(b). As the defect density is increased, the percolating cluster extends deeper into the sample until at some critical density it will touch the opposite face. Also, even for the same defect density and thickness, if the sample were made wider (corresponding to a larger device area), there is a finite probability of locally finding a cluster that does span the thickness. Thus, this rather simple model accounts nicely for all the statistical features of oxide breakdown including thickness and area dependence.

Fig. 6 shows the cumulative failure $(F)$ distributions for $N^{\mathrm{BD}}$ calculated from this model [33] along with experimental data [24]. The data are capacitance voltage (CV) measurements of the interface state density. Although it was stated earlier that the bulk traps control breakdown, the interface state generation parallels the bulk defects. For both theory and experiment, the slope of the failure distribution is an increasing function of sample thickness and the curves shift with thickness. The slope of the failure distribution is an important parameter used in reliability estimations, as will be discussed in a later section of this paper.

The measured critical defect density at breakdown is shown in Fig. 7 as a function of stress voltage, with thickness as a parameter [77]. Each point in this figure represents the final defect

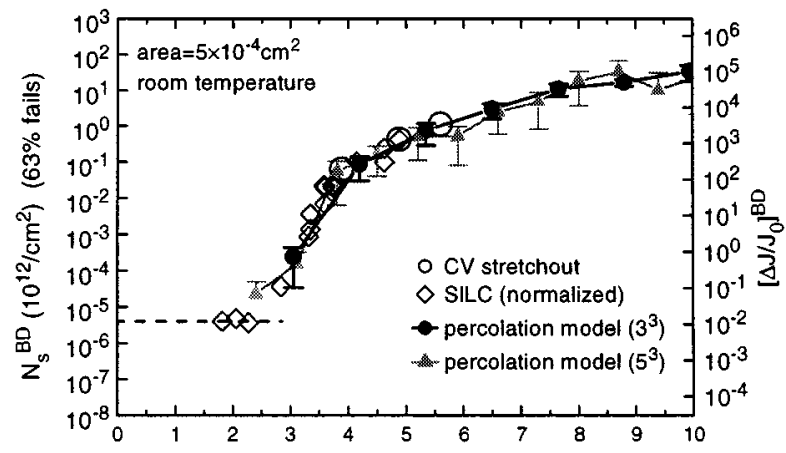

Fig. 8. Critical defect density for breakdown as a function of oxide thickness The CV and SILC data are normalized at $5 \mathrm{~nm}$. The fit to the percolation model is shown.

density extrapolated to breakdown for an individual sample. In addition to CV measurements (used for oxides with $t_{\mathrm{ox}} \geq 4$ $\mathrm{nm}$ ), this figure includes SILC data. In SILC measurements, the relative increase in current in the direct tunneling range below $3 \mathrm{~V}$ is expressed as $\Delta J / J_{0}$ where $J_{0}$ is the initial current density in the as-fabricated device. This quantity is proportional to the density of generated neutral electron traps [44]. Thus, $N^{\mathrm{BD}}$ from SILC measurements is in units of $\Delta J / J_{0}$. The critical defect density was taken as the value of $\Delta J / J_{0}$ immediately prior to the first soft or hard breakdown. However, for high fluence (i.e., near breakdown) and especially for low stress voltages, the SILC tends to saturate with increasing stress duration [44], [105]. This may limit the usefulness of SILC as a direct measure of $N^{\mathrm{BD}}$. The two vertical scales in Fig. 7 have been adjusted to match the average values obtained using the two measurement techniques on the 5-nm sample, from which we obtain [33] the relation $N_{s}\left(\mathrm{~cm}^{-2}\right) \approx 3 \times 10^{8} \Delta J / J_{0}$. This is consistent with the value found [44] for bulk the neutral traps, where $N_{n}\left(\mathrm{~cm}^{-2}\right) \approx 1.25 \times 10^{8} \Delta J / J_{0}$.

The thickness dependence is the dominant effect on $N^{\mathrm{BD}}$, but there may be some $V_{g}$ dependence as a second-order effect. The observed $V_{g}$ dependence may be partly attributed to the measurement technique, since CV and SILC data on the same 4-nm sample show different dependencies. In Fig. 8, we plot the average $N^{\mathrm{BD}}$ value as a function of $t_{\mathrm{ox}} \cdot N^{\mathrm{BD}}$ drops by a factor of $\sim 10^{5}$ from 6 to $3 \mathrm{~nm}$, and then reaches a plateau below 3 
$\mathrm{nm}$. These features are fully explained by a percolation model [33] shown by the filled symbols. The fit is described by two parameters, which are adjusted to give a consistent fit to both the data above $3 \mathrm{~nm}$ and the plateau below this thickness. The two parameters are the defect diameter (hopping distance) $a_{0}$ and the probability $(f)$ that a percolation path triggers destructive breakdown. For $t_{\mathrm{ox}}<a_{0}$, the percolation model predicts that the thickness dependence will vanish because only one "defect" is required to form a connecting path across the oxide. Therefore, the plateau region is $t_{\mathrm{ox}}<a_{0}$, and $N^{\mathrm{BD}}$ in the plateau corresponds to one active defect in the area of the sample. In terms of absolute defect density, using the SILC normalization discussed earlier the plateau value below $3 \mathrm{~nm}$ corresponds to a constant number equal to 2000 defects in the $5 \times 10^{-4} \mathrm{~cm}^{2}$ area of each sample, suggesting that each percolation path has a probability of about $10^{-3}$ of initiating a destructive breakdown event. In other words, only a fraction $f \sim 10^{-3}$ of the defects are "active" or "effective" in causing breakdown. This might result from the different energy levels of defects, or from their different physical or chemical nature. The idea that there exists some special subset of defects which trigger breakdown has been independently suggested from the electron microscopy observation of breakdown patterns [107] where it was found that the density of "weak spots" is $\sim 1 \%$ of the defect density at breakdown.

The value of the defect "size" obtained from the $N^{\mathrm{BD}}$ data is $a_{0} \approx 3 \mathrm{~nm}$. This is believed to correspond physically to the electrical sphere of influence of a point defect, e.g., a tunneling distance or trapping cross section. Other authors obtained smaller values for the defect diameter $(1.6-1.8 \mathrm{~nm})$ based on fitting to the distribution of breakdown times [31] or resistances [108].

One component of the percolation model is that $N^{\mathrm{BD}}$ is relatively independent of how the oxide is stressed. This has been the assumption whenever $N^{\mathrm{BD}}$ measurements at elevated voltage have been used to project oxide reliability down to operating conditions [104], [109]-[113]. Several experiments have supported this assumption, using various measurements of the defect density including interface states, trapped charge, and stress-induced leakage. [24], [32], [93], [114]-[118]. On the other hand, several groups have reported measurements showing a decrease in $N^{\mathrm{BD}}$ as the stress voltage is reduced using 3-5-nm oxides [77], [119]-[121]. This observation could have a significant effect on the reliability projections for such oxides.

In order to measure $N^{\mathrm{BD}}$ at lower voltage, i.e., closer to actual operating conditions, it has been necessary perform long-term reliability experiments on bonded chips. Other long duration stress experiments have been performed [91], [122] using thicker oxides. Fig. 9 shows SILC measurements performed for more than 1 year at $2.4 \mathrm{~V}$ using n-FETs with $t_{\mathrm{ox}}=2.2 \mathrm{~nm}$. The horizontal dotted lines in this figure indicate roughly the $10 \%$, median, and $90 \%$ values of $\Delta J / J_{0}$ at breakdown for the higher stress voltages. The $2.4-\mathrm{V}$ stress shows breakdown events occurring at statistically significant higher values of $N^{\mathrm{BD}}$. Fig. 10 shows the cumulative distributions for $N^{\mathrm{BD}}$ this sample. For stress voltage $\geq 2.8 \mathrm{~V}$, the $N^{\mathrm{BD}}$ distributions are nearly coincident, consistent with the assumption that $N^{\mathrm{BD}}$ is independent of $V_{g}$. For lower voltages, however, the distributions shift toward higher values of $N^{\mathrm{BD}}$.

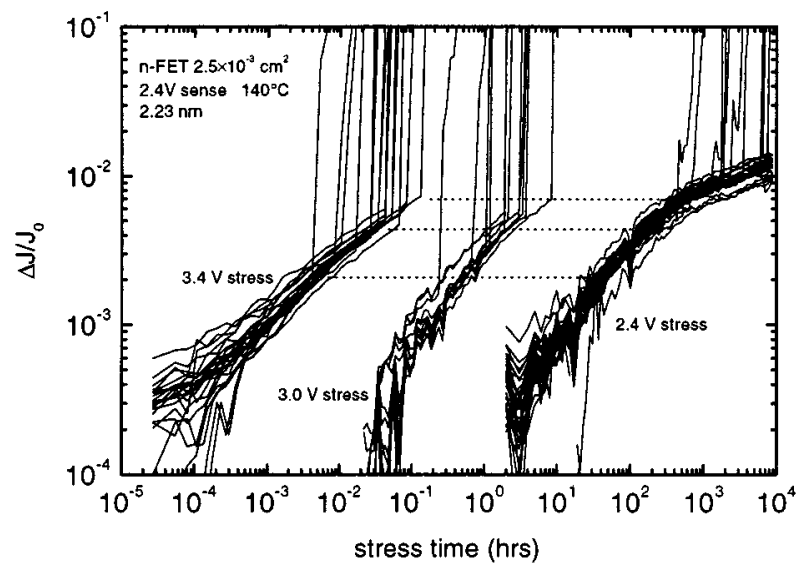

Fig. 9. Long-term stress data, showing SILC data to breakdown for three different stress voltages.

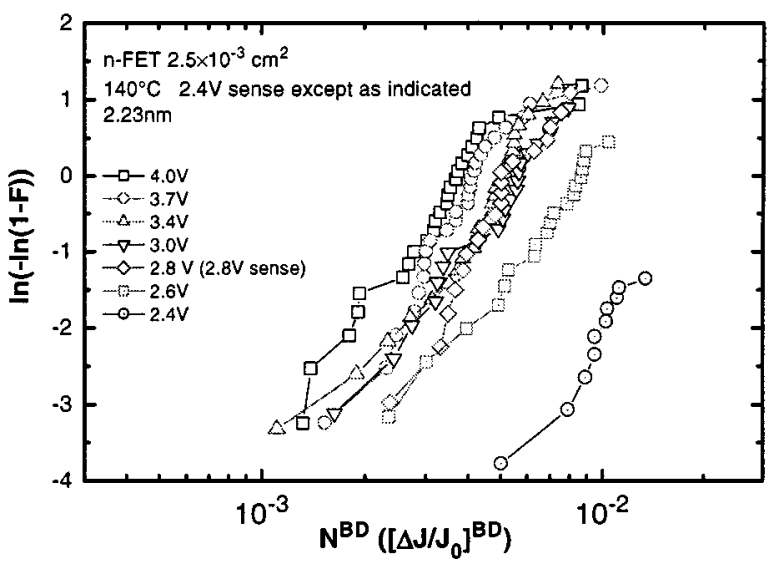

Fig. 10. Weibull plot of the critical defect density distributions at various stress voltages for 2.2-nm oxide.

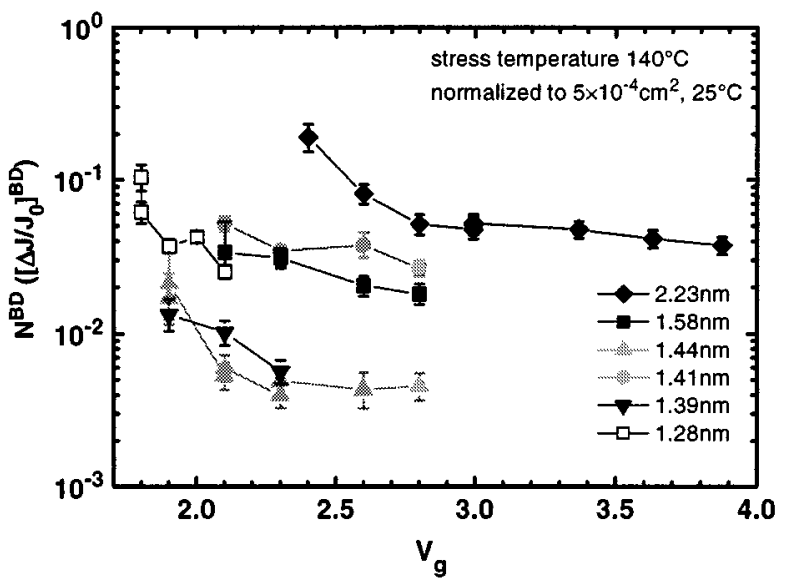

Fig. 11. $N_{\mathrm{BD}}$ versus stress voltage for various ultrathin oxides.

In Fig. 11 is plotted the characteristic (63rd percentile) values of $N^{\mathrm{BD}}$ versus the gate voltage during stress $\left(V_{g}\right)$ for the same sample as in Figs. 9 and 10 as well as some thinner oxide samples. The oxide thickness is estimated from the accumulation capacitance including quantum mechanical corrections, but it must be admitted that the quoted values are uncertain to at least $\pm 0.1 \mathrm{~nm}$. Error bars in $N^{\mathrm{BD}}$ reflect the statistical uncertainty, 


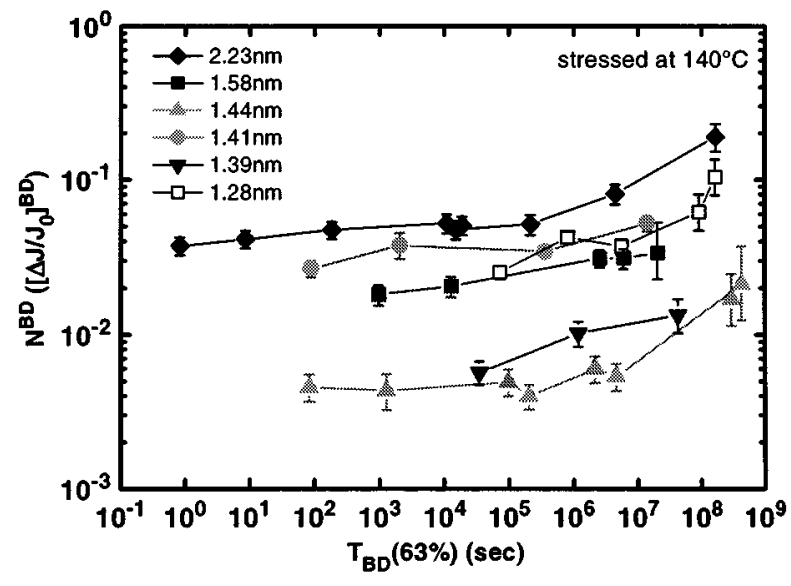

Fig. 12. $N_{\mathrm{BD}}$ versus stress time for various ultrathin oxides.

and in some cases at the lowest voltages only $\sim 10 \%$ of the samples had failed and therefore $N^{\mathrm{BD}}$ is estimated using the initial fail data and the statistical distributions from higher voltages. In this figure, the $N^{\mathrm{BD}}$ values have been normalized to a reference area of $5 \times 10^{-4} \mathrm{~cm}^{2}$ using Weibull statistics, taking the Weibull slope from data such as those in Fig. 10. The Weibull slope (discussed in more detail later) and $N^{\mathrm{BD}}$ are only weakly dependent on $t_{\mathrm{ox}}$ in this range. It can be observed that $N^{\mathrm{BD}}$ tends to increase with reducing $V_{g}$. In Fig. 9, it can be seen that the SILC tends to saturate at long times, i.e., the increase in SILC becomes sublinear in time for low voltage. A tendency for SILC to exhibit a saturating behavior at low stress voltage has been reported previously [44], [105]. In spite of this tendency, the ultrathin oxide samples stressed at lower voltage reach a higher average value of $\Delta J / J_{0}$ before breakdown. Therefore, while the measured $\Delta J / J_{0}$ may underestimate $N^{\mathrm{BD}}$, the observed increase at low voltage is real and correlates with an increase in charge-to-breakdown at low voltage [123].

Although the data in Fig. 11 show a trend for $N^{\mathrm{BD}}$ to increase as $V_{g}$ is lowered, there does not appear to be a universal behavior as a function of $V_{g}$. For example, for the 2.23-nm oxide, $N^{\mathrm{BD}}$ starts to increase below $2.8 \mathrm{~V}$, while for the 1.44-nm oxide the increase in $N^{\mathrm{BD}}$ is not seen until $V_{g}$ is reduced below $\sim 2.2 \mathrm{~V}$, and the 1.28-nm oxide shows no increase in $N^{\mathrm{BD}}$ even for $V_{g}$ as low as $1.9 \mathrm{~V}$. In Fig. 12, the same data have been replotted as a function of the breakdown times $\left(T_{\mathrm{BD}}\right)$ instead of the stress voltage. $T_{\mathrm{BD}}$ is a function of $V_{g}, t_{\mathrm{ox}}$, and device area. In this figure, $T_{\mathrm{BD}}$ is the projected value to $63 \%$ failure for cases where not all samples have reached breakdown. Note that, in this figure, $N^{\mathrm{BD}}$ is again normalized to a constant reference area, but $T_{\mathrm{BD}}$ is not normalized, i.e., the $x$-axis values reflect the actual time under stress. Here we see that the data show a universal trend, wherein $N^{\mathrm{BD}}$ starts to increase for stress times longer than about $10^{6} \mathrm{~s}$ ( $\sim 10$ days). The long stress times necessary to see this effect would preclude its observation in most experiments. For the 1.28-nm oxide at $1.8 \mathrm{~V}$ (open squares), devices with two different areas were tested, which when plotted against $V_{g}$ result in different values of $N^{\mathrm{BD}}$ even after area normalization (Fig. 11). However, smaller area devices have a longer lifetime, which accounts for the higher value of $N^{\mathrm{BD}}$ as shown in Fig. 12.
A voltage-dependent $N^{\mathrm{BD}}$ could arise from several sources. The percolation path, which has been modeled in zero field [31], [33] to obtain the fit shown in Fig. 8, could be weakly field-dependent. The formation of the percolation path, i.e., the generation of new defects, could depend on the local field produced by the other defects [124], [125]. This would lead to more directed paths at higher voltage, so that the average defect density to form a connecting path across the sample would be reduced. However, according to the idea that the stress time, not the voltage, is responsible for the increase in $N^{\mathrm{BD}}$, the data may be interpreted in terms of the defect "effectiveness" (the fraction $f$ of "active" defects) which was introduced [33], [107], [121] to describe the probability that a defect, or percolation path, can trigger breakdown. For long stress times, a greater defect density is required to trigger breakdown, contrary to the assumption that $N^{\mathrm{BD}}$ should be independent of the stress condition. The observation of reduced defect effectiveness for very long duration stress experiments may imply that defects undergo a slow relaxation that reduces their ability to participate in breakdown. It must be emphasized, however, that the final runaway stage of destructive breakdown, whereby a percolation path leads to catastrophic failure, is still not fully understood.

\section{Defect Generation Rate}

Extensive work in the 1980s revealed the existence of the so-called "2-eV trap creation threshold" (electron energy measured with respect to the bottom of the $\mathrm{SiO}_{2}$ conduction band) for defect creation by hot electrons in $\mathrm{SiO}_{2}$ [23]. For thick oxides, electrons in the conduction band obtain this average energy for oxide fields greater than about $2-4 \mathrm{MV} / \mathrm{cm}$. In the thin oxides which are of current interest, for which electron transport is ballistic or quasi-ballistic, the threshold corresponds to a gate voltage of about $5 \mathrm{~V}$ for Fowler-Nordheim tunneling through the $3-\mathrm{eV}$ potential barrier at the $\mathrm{Si} / \mathrm{SiO}_{2}$ interface. For voltages below threshold, it was shown [24] that the defect generation rate depends only on the absolute value of the gate voltage $\left(V_{g}\right)$, independent of substrate or gate doping or polarity. Recent work using the SILC to measure $P_{g}$ has given the somewhat surprising result that $2 \mathrm{eV}$ is not a hard threshold [25], [119]. Instead, there is a subthreshold trap generation process that decreases exponentially below $5 \mathrm{~V}$. This is shown in Fig. 13, where the SILC and CV data have again been normalized to each other using the same relation as described above for $N^{\mathrm{BD}}$.

Fig. 13 includes data from a variety of oxide thicknesses and processes, including "standard" thermal $\mathrm{SiO}_{2}$ grown in $\mathrm{O}_{2}$, oxides grown in $\mathrm{N}_{2} \mathrm{O}$, and oxides grown on $\mathrm{N}_{2}$-ion-implanted $\left(\mathrm{N}_{2}\right.$-I/I) substrates, indicating the relative insensitivity of $P_{g}$ to the oxidation process. $P_{g}$ is also observed to be independent of stress voltage polarity. For each oxide thickness, it is possible to measure $P_{g}$ over only a limited range of $V_{g}$, in order to keep the measurements within an experimentally accessible time scale. Plotting overlapping ranges of $V_{g}$ obtained using oxides of different thickness shows a universal exponential behavior of $P_{g}$ as a function $V_{g}$, independent of $t_{\mathrm{ox}}$. Using substrate hot electron (SHE) and channel hot electron (CHE) stress, it is possible to obtain much greater hot electron flux at the interface, permitting measurements of $P_{g}$ at low electron energy even for thick 


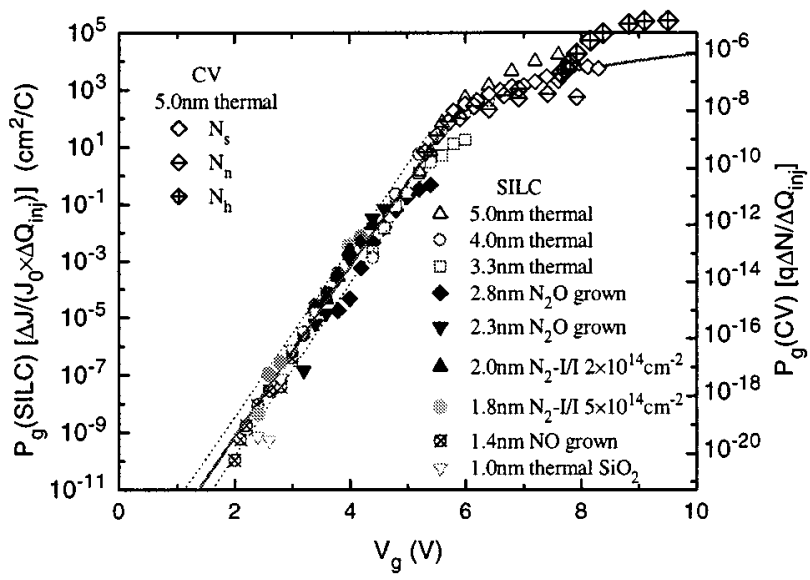

Fig. 13. Defect generation probability measured from SILC $\left(P_{g}\right.$ (SILC)) and CV stretch-out $\left(P_{g}(\mathrm{CV})\right.$ and $\left.N_{S}\right)$, and electron and hole trapping rates $\left(N_{n}\right.$ and $N_{h}$, respectively), as a function of stress voltage for various oxides.

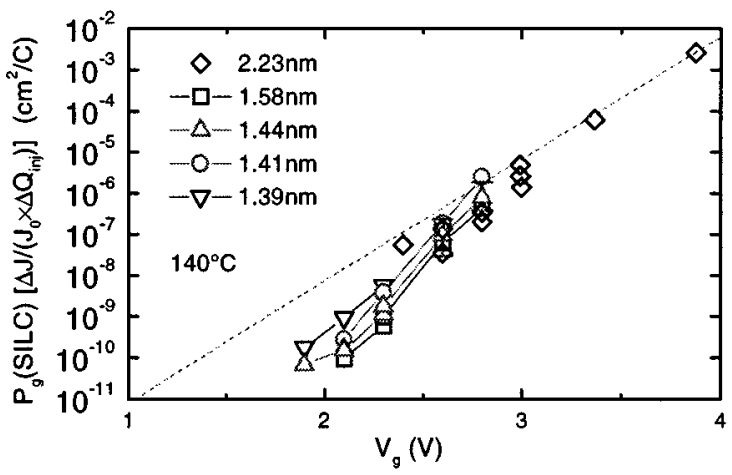

Fig. 14. Detail of $P_{g}$ data from long-term stress experiments, showing deviation from purely exponential behavior.

samples. Using these techniques, it has been shown [25], [126], [127] that defect generation by hot electrons impinging on the substrate-oxide interface follows the same dependence on energy as that from Fowler-Nordheim injection through the oxide, and that the exponential $V_{g}$ dependence extends at least as low as $1.2 \mathrm{~V}$ [127].

A remarkable and unexpected feature of the data of Fig. 13 is that there is no hard threshold voltage below which the generation rate drops faster than the exponential trend. In particular, there is no large discontinuity or change in slope at the transition from Fowler-Nordheim to direct tunneling at about $3 \mathrm{~V}$. This is contrary to earlier suggestions [128], [129] that breakdown would be strongly suppressed in the direct tunneling regime and strongly suggests that the relevant energy scale is the electron energy in the anode, which is the poly-silicon gate or the silicon substrate depending on injection polarity. Although the rate of defect generation decreases approximately exponentially with supply voltage below a threshold voltage of about $5 \mathrm{~V}$ for hot electron-induced hydrogen release, detailed measurements [123], [127] have found an inflection in the voltage dependence of $P_{g}$ between 2-3 V. This is shown in Fig. 14. Taken together, the complete measurements of $P_{g}$ and $N^{\mathrm{BD}}$ can explain in detail the voltage and thickness dependence of $Q_{\mathrm{BD}}$ in ultrathin gate oxides, shown in Fig. 15(a).
In addition to the interface state $\left(N_{s}\right)$ and bulk electron trap $\left(N_{n}\right.$ and SILC) densities, Fig. 13 also includes data on hole trapping $\left(N_{h}\right)$ showing the anode hole injection threshold at $\sim 7.5 \mathrm{~V}$. [22] The offset between this threshold and the lower trap creation threshold clearly establishes the subthreshold tail as being related to the trap creation (hydrogen release) process and not anode hole injection.

Measured over a sufficiently wide range of stress conditions, neither $T_{\mathrm{BD}}$ nor $Q_{\mathrm{BD}}$ will obey any simple "law" such as exponential dependence on $E, 1 / E$, or $V_{g}$, as has been commonly assumed in reliability extrapolations. Without the simplifying assumptions of a voltage-independent $N^{\mathrm{BD}}$ and a purely exponential voltage dependence of $P_{g}$, it becomes more difficult to extrapolate reliability to operating voltage. The steeper $V_{g}$ dependence of $P_{g}$ between 2-3 V can easily lead to an overly optimistic $T_{\mathrm{BD}}$ projection if data are limited to this range. Likewise, projection of data from higher voltages without knowing the complete $V_{g}$ dependence may lead to more pessimistic projections. Moreover, according to the idea that $N^{\mathrm{BD}}$ increases for long stress duration, breakdown is a time-dependent as well as voltage-dependent phenomenon. Care must be taken when interpreting $Q_{\mathrm{BD}}$ or $T_{\mathrm{BD}}$ data to separate the voltage- and timedependent effects.

\section{LIFETIME}

Although $Q_{\mathrm{BD}}$, defined as the time-integrated current density that flows through the oxide until breakdown occurs, is the physically meaningful quantity, the quantity of interest for an electronic component is the failure rate, which can be derived from the lifetime or time-to-breakdown, $T_{\mathrm{BD}}$. For constant voltage stress, this is related to $Q_{\mathrm{BD}}$ by the relation

$$
\int_{0}^{T_{\mathrm{BD}}} J d t=Q_{\mathrm{BD}}
$$

where $J$ is the instantaneous value of the current density. For thin oxides, the current is nearly constant until breakdown (in marked contrast to thicker oxides, where electron trapping and/or hole trapping cause large changes in the current during stress), therefore

$$
T_{\mathrm{BD}}=Q_{\mathrm{BD}} / J
$$

The tunnel current density increases exponentially with decreasing oxide thickness [104]. Therefore, from (4), $T_{\mathrm{BD}}$ decreases exponentially with decreasing $t_{\mathrm{ox}} \lesssim 3 \mathrm{~nm}$ even though $Q_{\mathrm{BD}}$ [Fig. 15(a)] is only slightly thickness-dependent in this range due to the weak thickness dependence of $N^{\mathrm{BD}}$. The measured $T_{\mathrm{BD}}$ data are shown in Fig. 15(b). This implies a rapidly diminishing margin for reliability as device dimensions are scaled. Fig. 15(b) also shows that data from different labs [112], [123], [130] are in reasonable agreement, taking into account the differences in oxide thickness, indicating little dependence on processing for state-of-the-art facilities.

It is commonplace in the microelectronics industry to specify an operating life of ten years, i.e., to guarantee a specified (usually small) failure rate over a 10 -yr period. First the $T_{\mathrm{BD}}$ data 


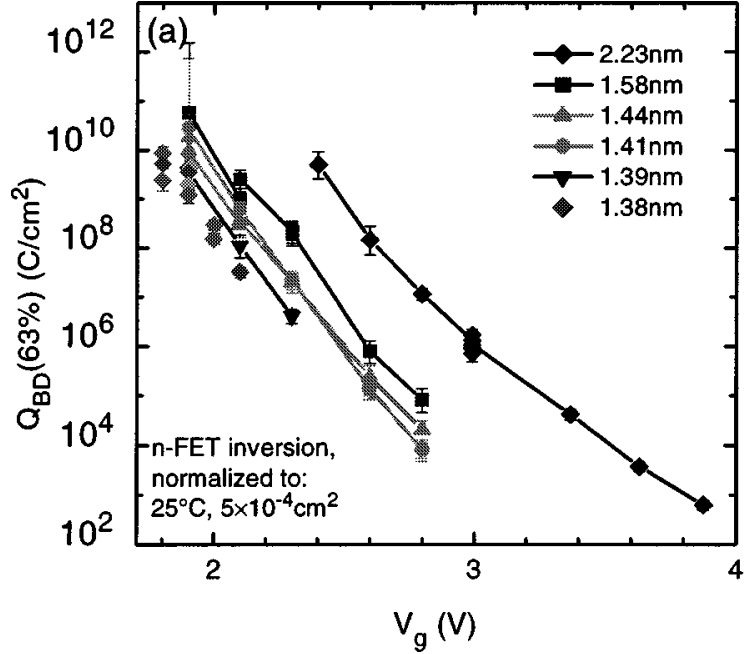

(a)

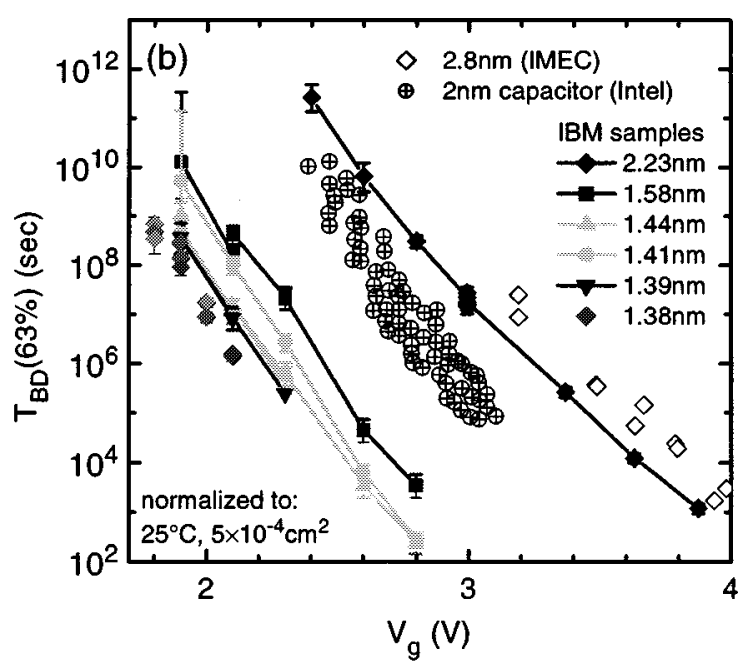

(b)

Fig. 15. (a) $Q_{\mathrm{BD}}$ data from long-term stress experiments on ultrathin oxides. (b) $T_{\mathrm{BD}}$ data from long-term stress experiments, and published data from other labs. All data have been scaled to a reference area using Weibull statistics and to room temperature.

such as those in Fig. 15(b) must be projected from the high percentiles where experimental data are collected to low percentiles desired for the product failure rate. Second, a lifetime correction must be made from the small-area test structures to the total gate oxide area of a chip. Both of these projections depend on the shape of the breakdown distribution, which must be known for accurate reliability estimates.

The statistics of gate oxide breakdown are described using the Weibull distribution [5], [31], [131]-[134]

$$
F(x)=1-\exp \left[-(x / \alpha)^{\beta}\right]
$$

which is an extreme-value distribution in $\ln (x)$ and is appropriate for a "weakest-link" type of problem. Here $F$ is the cumulative failure probability, i.e., the population fraction failed by age $x$, where $x$ can be either charge or time. The characteristic life $\alpha$ is the 63.2th percentile, and $\beta$ is called the slope parameter or Weibull slope. Plotting

$$
W \equiv \ln [-\ln (1-F)]
$$

against $\ln (x)$ yields a straight line with slope $\beta$ (see Fig. 16).

Gate oxide failure is a weakest-link type of problem because failure of the whole chip is defined by the failure of the first individual device, and a device fails if any small portion of the gate area of the device breaks down. From elementary statistics, if the probability of any one unit failing is $p$ then the probability of any one of $N$ independent units failing is

$$
F=1-(1-p)^{N}
$$

so that

$$
\ln [-\ln (1-F)]=\ln N-\ln [-\ln (1-p)] .
$$

The Weibull plot [(6)] thus has the extremely useful property that if the area is increased by a factor $N$ then the curve shifts vertically by $\ln (N)$. Fig. 16 illustrates this effect schematically. If the desired low failure rate is $F_{\text {chip }}$ over the product lifetime $T_{\text {life }}$ for the total gate area $A_{\mathrm{ox}}$ on the chip (point indicated by

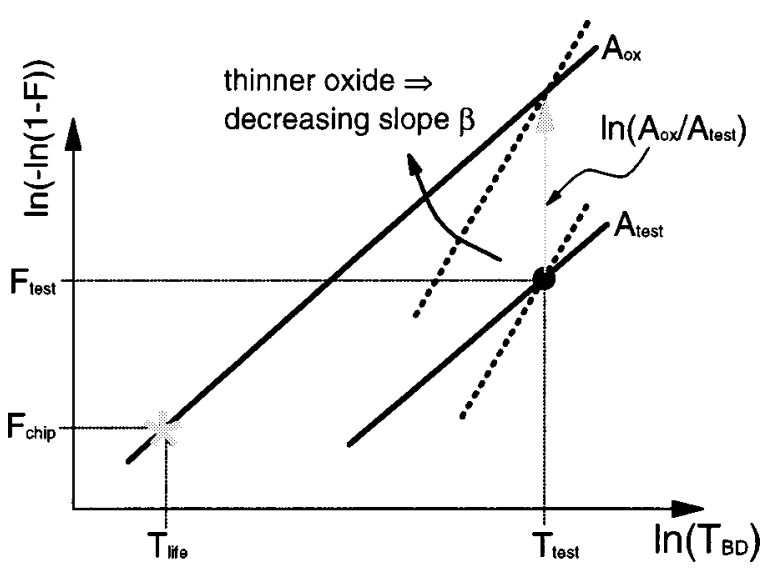

Fig. 16. Procedure for extrapolation to small failure rate and chip area, illustrating the effect of Weibull $\beta$ on area scaling and failure rate projection.

the asterisk in Fig. 16), this is equivalent to a higher failure rate $F_{\text {test }}$ in time $T_{\text {test }}$ on the test structures with area $A_{\text {test }}$, where from Fig. 16 we can obtain

$$
\begin{aligned}
\frac{T_{\text {life }}}{T_{\text {test }}} & =\left(\frac{A_{\text {test }}}{A_{\text {ox }}}\right)^{1 / \beta}\left(\frac{\ln \left(1-F_{\text {chip }}\right)}{\ln \left(1-F_{\text {test }}\right)}\right)^{1 / \beta} \\
& \approx\left(\frac{A_{\text {test }}}{A_{\text {ox }}} \frac{F_{\text {chip }}}{F_{\text {test }}}\right)^{1 / \beta} .
\end{aligned}
$$

The appropriate $\beta$ to use in (9) is the one corresponding to the projected operating voltage. The assumption is generally made that $\beta$ is independent of $V_{g}$. This equation is used to scale measured breakdown times to the expected product lifetime, or equivalently to estimate the chip failure rate from test structure measurements. Since $F_{\text {chip }}<F_{\text {test }}$ and typically $A_{\text {test }}<A_{\mathrm{ox}}$, then $T_{\text {test }}>T_{\text {life }}$ by this equation, therefore it is always necessary to measure the test structure under accelerated stress conditions (voltage and temperature). Understanding the voltage dependence is the major reason for investing the time to obtain long-term stress data as shown in Fig. 15, and is the reason so much attention is paid to the physical model for trap generation and breakdown, as discussed above [135], [136]. 
There is no guarantee in practice that actual failure distributions will be perfectly linear on a Weibull plot, and other distributions could in principle be more appropriate [133]. Nonetheless, a study of 900 samples [135] clearly supported the Weibull distribution down to a failure rate of $10^{-3}$. In the simplest case, the presence of multiple failure modes such as extrinsic failure caused by processing defects will lead to bi- or multi-modal distributions with varying slope. A subtler problem can arise from nonuniformity of $t_{\mathrm{Ox}}$, which will lead to variations in $Q_{\mathrm{BD}}$ because of the thickness dependence of the critical defect density and will cause further variation in $T_{\mathrm{BD}}$ because of the thickness dependence of the tunnel current. This will lead to distortion and curvature of the Weibull plot [137], [138]. Such issues can seriously complicate the otherwise rather straightforward projections. Curvature in the Weibull plot usually indicates a problem such as extrinsic failure modes or nonuniformities that need to be dealt with before the data can be reliably interpreted.

From the previous discussion, it can be seen that an important parameter for reliability projections is the Weibull slope $\beta$. A key advance was the realization that $\beta$ is a function of $t_{\mathrm{ox}}$, becoming smaller as $t_{\mathrm{ox}}$ decreases [24], [31], [33]. The thickness variation of the Weibull slope of the $Q_{\mathrm{BD}}$ distribution stems from the properties of $N^{\mathrm{BD}}$ and is a statistical property of the percolation model for $N^{\mathrm{BD}}$. It is an intrinsic property of the breakdown of ultrathin oxides and does not imply that the breakdown mechanism is changing. A smaller $\beta$ means greater sensitivity to the area and failure rate extrapolations and gives more pessimistic projections via (9), so this is a crucial issue for predicting breakdown of ultrathin oxides. It is often difficult to obtain an accurate value of $\beta$ from a direct measurement of the failure distribution, because of thickness variations and statistical uncertainty [139]. In order to circumvent these difficulties, $\mathrm{Wu}$ [135], [140] adopted the area scaling relation of (9) in order to obtain accurate values.

Fig. 17 shows schematically how the failure rate and area projections each reduce the maximum operating voltage allowable for a particular reliability specification. The effects are greater for thinner oxides, because of the lower value of $\beta$. In addition, the operating temperature must be taken into account, since $T_{\mathrm{BD}}$ and $Q_{\mathrm{BD}}$ are strongly temperature-dependent especially for $t_{\mathrm{ox}} \lesssim 3 \mathrm{~nm}$ [77], [141], [142].

\section{FUnCTIONAL RELIABILITY}

The preceding discussion has shown how the reliability margin for gate oxide breakdown has been drastically reduced as a consequence of device scaling. Published models [69], [104], [111], [112], [123] have indicated that, for the oxides used in the past with $t_{\mathrm{ox}} \gtrsim 3-4 \mathrm{~nm}$, intrinsic gate oxide reliability has probably not been a real issue. However, the time is near when it will no longer be possible to meet reliability specifications for ultrathin gate oxides, although the exact point when this will happen is the subject of considerable debate. At present, predicted reliability "limits" for the gate oxide thickness range from less than $1.5 \mathrm{~nm}$ [69] to $2.8 \mathrm{~nm}$ [111], [112]. Even if the smallest estimates were correct, the latest international roadmap for the industry [143] anticipates that $t_{\mathrm{ox}} \sim 1 \mathrm{~nm}$ will be needed by 2005 for $60-70-\mathrm{nm}$ gate

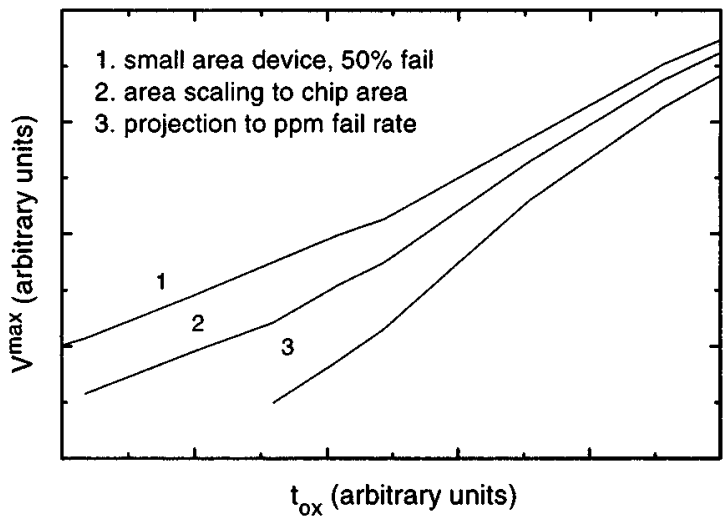

Fig. 17. Schematic illustration of the effect of area and failure rate projections on the maximum operating voltage as a function of oxide thickness.

lengths (90-nm lithography node) operating at $\sim 1 \mathrm{~V}$ in order to meet the desired performance targets. One way this might be achieved is by replacing the $\mathrm{SiO}_{2}$ with another dielectric with a higher dielectric constant, such as metal oxides, metal silicates, and epitaxial perovskites. This avenue is being aggressively pursued but faces large hurdles, as neither the materials science nor the electrical properties of these materials are understood nearly as well as for $\mathrm{SiO}_{2}$. A more conservative approach is to used nitrided oxides, which have shown somewhat improved reliability characteristics compared to pure $\mathrm{SiO}_{2}$ [144], [145].

Up to now, the definition of oxide breakdown has generally been the first event marked by a discrete jump in leakage current. Some researchers have pointed out, however, that often the magnitude of this jump is not great enough to completely destroy the functionality of a transistor, much less that of an entire circuit [146]-[148]. It has been suggested that the definition of breakdown which we have been using, although physically a correct signature of the formation of a conducting path across the insulator, may not be the appropriate practical criterion for product reliability specification. Most work up to now has focused on the understanding of the defect generation leading to breakdown. If product reliability is to be assured, it will be necessary to focus more attention on understanding the nature of the conduction after breakdown [149]-[159] and on the impact of this conduction on device and circuit functionality.

\section{A. Soft Breakdown}

The so-called "soft" breakdown (SBD) or "quasi-breakdown" was first reported [160]-[162] in 3-4-nm oxides subjected to either constant-current [161], [162] or constant-voltage [160] stress. After first showing a uniform degradation in the form of SILC, the oxides exhibited a sudden jump to a fluctuating (noisy) current with high leakage at low voltage. The SBD phenomenon has recently been thoroughly reviewed [158] and so we will only highlight certain aspects here.

The fact that the $I-V$ characteristic after SBD merges with the prebreakdown Fowler-Nordheim characteristic at higher voltages distinguishes this mode from the destructive, or "hard" breakdown (HBD). The latter type is probably a result of thermal damage [158] when sufficient energy is deposited during the breakdown transient [107], [163]-[174]. There is probably some confusion in the literature over the precise 
characterization of breakdown events as "soft" or "hard" because of the lack of a precise definition and because for some experimental conditions the detection of one or the other breakdown mode may be difficult. For example, when testing a large-area structure or a very thin oxide where the initial current is larger than the breakdown current, a "soft" event could be missed, or a "hard" event could be interpreted as soft. Initially, it was argued [175] that SBD is a precursor of HBD, i.e., that an SBD spot will become hard after some time. This view was not consistent with a later statistical analysis of data [176] showing that the distribution of breakdown times is independent of whether they are soft or hard, and that an HBD which occurs after an SBD is a random event uncorrelated with the first SBD spot. It was also shown that SBD and HBD follow similar area dependence [177] but the temperature dependence and voltage dependence were found to be different [178] or the same [179] in different laboratories. The similar light emission spectral characteristics of SBD and HBD [180] also support the idea that they are related phenomena, differing only in the size of the breakdown spot. This conclusion has been questioned in turn by a different analysis of data [181] finding subtle differences in the parameters describing both the statistical distributions and the voltage dependence of SBD and HBD.

Soft breakdown is the most common mode for a constant-current stress, while hard breakdown may occur during constant-voltage stress [182]. The soft breakdown is a localized spot, which can be shown by measuring the area dependence [153], [158], [160], [161], [181] or by viewing the optical emission [17], [161], [181], [183]. The size of this spot is $10^{-14}$ to $10^{-12} \mathrm{~cm}^{2}$ [158]. Several soft breakdown events, occurring in different spots, may sometimes be seen in large-area devices prior to a hard breakdown [153], [155], [158], [160], [175], [184]. In addition, the SBD current voltage $(I-V)$ characteristic is independent of the oxide thickness at least down to $3 \mathrm{~nm}$, within a band of observed curves [153], [155], [158], [185]. In contrast to the "hard" breakdown (HBD) which shows a roughly linear (ohmic) $I-V$ characteristic of resistance $\sim 10 \mathrm{k} \Omega$ if the damage region remains localized and does not propagate, [107], [158] the SBD $I-V$ characteristic is a power-law with an exponent of 3-6, [149], [150], [158], [186] although it maybe better described by an exponential voltage dependence [159]. The SBD voltage dependence can be explained by a quantum point contact model [151], [153]-[156], [158], [159], although other models have been proposed as well [149], [150], [187]-[190]. The point contact model can account for both SBD and HBD within a single framework as two limiting cases, depending only on the lateral size of the breakdown spot which determines the energies of the subbands in the conduction path [158].

Simultaneous with the jump in gate current after SBD is a large increase in the substrate hole current (for the case of n-FETs biased in inversion) [161], [189]-[192] and the p-channel hole tunneling current (for p-FETs in inversion) [174], [188]-[190]. For the n-FET case, this was attributed to valence band tunneling or back-injection of holes generated in the anode, but a recent study found that the spectral characteristics of the light emission from breakdown spots is similar to that generated by hot carriers in the channel of a MOSFET,

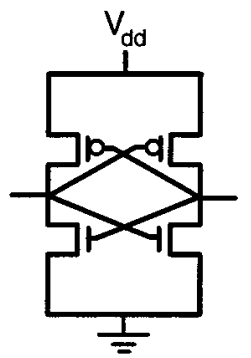

Fig. 18. Basic SRAM storage cell.

suggesting that the substrate current is dominated by impact ionization in the space charge region of the channel near the breakdown spot [180].

\section{B. Device Breakdown}

It was suggested early on [161] that the SBD leakage at low voltage would be too large for device applications and therefore SBD should be regarded as oxide failure. In addition, the appearance of large fluctuations in the direct tunneling current led to the consensus that SBD should be identified as the dielectric breakdown [162], [193]. This view was not questioned until others [146] claimed that gate current noise was the only detectable effect of SBD on n-FETs fabricated with a 2-nm gate oxide and that there was no correlated degradation in either the threshold voltage $\left(V_{T}\right)$ or transconductance $\left(g_{m}\right)$. It was therefore suggested that SBD would not cause device or circuit failure in many applications [146]. However, Pompl et al. [194] and others [135], [138], [195], [196] later showed that SBD will cause a significant increase in the transistor-off current if the breakdown spot is in the drain region, which is increasingly likely in short-channel devices. This will be referred to as "device breakdown." In a study of the channel length- and width-dependence of device breakdown in n-FETs stressed at 4.1 V, Wu [135], [177] showed that, for short-channel devices (0.2- $\mu \mathrm{m}$ channel length), the distribution of oxide breakdown times for the first breakdown event coincides with the distribution of device breakdown times, whereas for longer channels (10 $\mu \mathrm{m})$ the device breakdown occurs some time after the first soft breakdown. After a "hard" breakdown, the device is clearly nonfunctional by any ordinary criterion, exhibiting a negative drain current when the gate-to-drain leakage exceeds the normal transistor on current [194]. However, even a hard breakdown may not completely destroy circuit functionality: Kaczer et al. [197] showed that in some cases a circuit may be able to survive an oxide breakdown that previously would have been assumed to be catastrophic.

In a CMOS circuit, the stress conditions on a transistor gate are not the same as the typical experimental conditions used to study reliability. A circuit does not usually subject a gate to either a constant-voltage or a constant-current stress, which are the two types of stress typically employed, but rather to a current-limited stress in which the current through a breakdown spot is limited by the saturation on-current (Idsat) of a complementary transistor in series [172]. Fig. 18 shows the situation for an SRAM cell consisting of two cross-coupled inverters. Not all circuits will fall into this category, for example some gates 


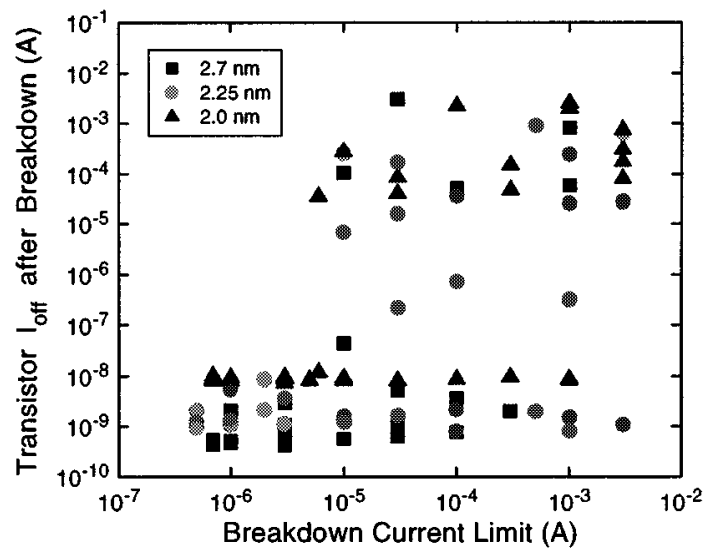

Fig. 19. The n-FET off current ( $\left.I_{\text {orf }}\right)$ after breakdown versus the value of the breakdown current limit.

will be driven by a low-impedance clock, but SRAM occupies a large fraction of the area of many chips and therefore it is a useful place to begin an investigation of circuit reliability. In the SRAM cell, the gate oxides are not connected directly to the power supply. The n-FET gates are connected via p-FETs, and the $\mathrm{p}$-FET gates via n-FETs. Compared to a typical constant voltage source, the small transistor in series has much higher impedance, a much lower current capability, and a lower capacitive loading. To simulate the stress that gate oxides experience during circuit operation, Linder [172] purposely limited the post-breakdown current using the compliance setting of a voltage source or by inserting a transistor between the voltage source and device under test. The compliance level could be set to emulate the saturation current of the drive transistor. In order to prevent this from being a simple constant current stress, the initial stress current must be significantly less than the current limit, necessitating the testing of small area devices.

The n-FET off current $\left(I_{\text {off }}\right)$ after breakdown is plotted versus the value of the current limit in Fig. 19. For a current limit less than $\sim 10 \mu \mathrm{A}$, the off current remains small after oxide breakdown, while higher current limits resulted in a wide range of $I_{\mathrm{off}}$ values [172]. Thus it appears that small current limits (corresponding to very small p-FET drivers) halt the breakdown event such that the transistors may still be operational. Toriumi [173], [174] similarly showed that increasing the circuit time constant, by inserting an inductive impedance in series with the device, will reduce the severity of the breakdown, without affecting the breakdown time, and Lombardo et al. have observed that the breakdown becomes softer at low inversion layer density [198]. This shows that the nature of the breakdown spot is influenced by the circuit environment of the device, which in turn may affect the overall projected reliability of a circuit.

Fig. 20 shows the post-breakdown conduction measured at $V_{g}=1.5 \mathrm{~V}$ as a function of the stress compliance level [172]. Again there is a transition at about $10^{-5}$ A compliance, corresponding to a series resistance of $\sim 10^{5} \Omega$. Below this value, the n-FET leakage remains below $10^{-7} \mathrm{~A}$, but for higher current limits the leakage increases more rapidly. The compliance level of $10^{-5}$ A corresponds to Idsat of a small p-FET, such as might be used in an SRAM cell. For fast logic circuits, p-FET Idsat values of $\sim 10^{-4} \mathrm{~A}$ are likely, in which case the n-FET

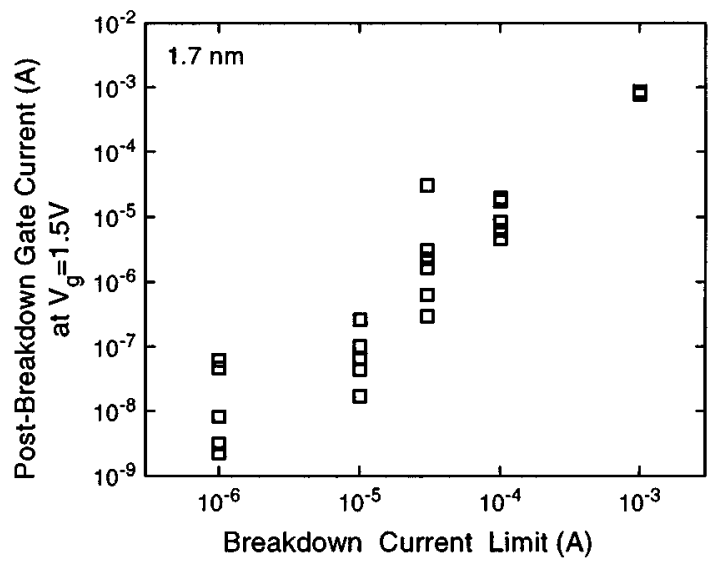

Fig. 20. The post-breakdown conduction measured at $V_{G}=1.5 \mathrm{~V}$ as a function of the stress compliance level.

post-breakdown leakage will average $\sim 10^{-5} \mathrm{~A}$. At this level of leakage, the voltage drop across the p-FET channel in Fig. 18 after n-FET oxide breakdown is expected to be $\sim 0.1 \mathrm{~V}$. This amount of voltage droop can probably be tolerated by most logic circuits. However, there is a distribution of post-breakdown leakage levels [108], [156], [158], [170], [171], [186] so it is likely that some circuit failures will occur as a result of oxide breakdown. The overall effect of SBD on circuit performance is still an open question since many different circuit elements are used in practice and some may be more sensitive than others to noise and voltage margins.

Even if devices survive after an initial breakdown event, the subsequent stress on the damaged oxide can lead to erratic behavior and a progressive degradation of the device characteristics [135]. Thus, it would be premature to disregard oxide breakdown as a factor in circuit reliability. Much more research will be needed in order to formulate a complete methodology for the quantitative prediction of device and circuit reliability.

\section{CONCLUSION}

In this paper, we have reviewed the physics and statistics of dielectric wearout and breakdown in ultrathin $\mathrm{SiO}_{2}$-based gate dielectrics. Electrons or holes tunneling through the gate oxide generate defects until a critical density is reached and the oxide breaks down. A percolation model can explain the critical defect density and the thickness dependence of the Weibull slope. Some fundamental aspects are still unknown, e.g., the microscopic nature of the defects and what factors determine how a percolation path leads to breakdown. The rate of defect generation decreases approximately exponentially with supply voltage, below a threshold voltage of about $5 \mathrm{~V}$ for hot electron-induced hydrogen release. There is strong evidence for the role of hydrogen in defect creation, but other models, specifically the thermochemical and AHI models, are also in widespread use. Some aspects of these models are not in accord with certain experimental data, and so more experimental and theoretical work will be required for a complete understanding.

The direct tunneling current increases exponentially with decreasing oxide thickness, leading to a decreasing time-to-breakdown and a diminishing margin for reliability as device dimen- 
sions are scaled. Estimating the reliability of the dielectric requires an extrapolation from the measurement conditions (e.g., higher voltage) to operation conditions. Because of the diminished reliability margin, it has become imperative to try to reduce the error in this extrapolation. Long-term $(>1$ year) stress experiments are now being used to measure the wearout and breakdown of ultrathin $(<2 \mathrm{~nm})$ dielectric films as close as possible to operating conditions. From measurements over a wide range of stress conditions, we find that neither $T_{\mathrm{BD}}$ nor $Q_{\mathrm{BD}}$ will obey any simple "law" such as exponential dependence on $E, 1 / E$, or $V_{g}$, as has been commonly assumed in reliability extrapolations. Thus, it becomes more difficult to extrapolate reliability to operating voltage.

The nature of the electrical conduction through a breakdown spot will have a significant bearing on the degree to which the oxide breakdown affects device and circuit performance. The definition of oxide breakdown has generally been the first event marked by a discrete jump in leakage current. However, sometimes the magnitude of this jump may not be great enough to completely destroy the functionality of a transistor, much less that of an entire circuit. The "hardness" of breakdown is influenced by several factors, including the circuit in which the oxide is used. Conversely, different circuits will have various degrees of sensitivity to erosion of noise and voltage margins resulting from oxide breakdown, so more research is needed in order to develop a quantitative methodology for predicting the reliability of circuits.

\section{ACKNOWLEDGMENT}

The author would like to acknowledge and thank D. J. DiMaria for his advice and guidance during the past 15 years. The extensive data in Figs. 2, 7, 8, and 13, and many of the physical insights describing those data, are due to him. The author would also like to thank A. Vayshenker for the long-term stress data and B. P. Linder for the current-limited breakdown data and for helpful discussions.

\section{REFERENCES}

[1] R. H. Dennard, F. H. Gaensslen, H.-N. Yu, V. L. Rideout, E. Bassous, and A. R. LeBlanc, "Design of ion-implanted MOSFET's with very small physical dimensions," IEEE J. Solid State Circuits, vol. SC-9, pp. 256-268, 1974

[2] N. Klein, "The mechanism of self-healing electrical breakdown in MOS structures," IEEE Trans. Electron Devices, vol. ED-13, pp. 788-805, 1966.

[3] P. Solomon, "Breakdown in silicon oxide-A review," J. Vac. Sci. Technol., vol. 14, pp. 1122-1130, 1977.

[4] E. Harari, "Dielectric breakdown in electrically stressed thin films of thermal $\mathrm{SiO}_{2}$, , J. Appl. Phys., vol. 49, pp. 2478-2489, 1978.

[5] D. R. Wolters and J. F. Verwey, "Breakdown and wear-out phenomena in $\mathrm{SiO}_{2}$ films," in Instabilities in Silicon Devices, G. Barbottin and A. Vapaille, Eds. Amsterdam, The Netherlands: North-Holland, 1986, pp. 315-362.

[6] Z. A. Weinberg, "High-field positive charge generation and its relation to breakdown in a-SiO ${ }_{2}$," in The Physics and Technology of Amorphous $\mathrm{SiO}_{2}$, R. A. B. Devine, Ed. New York: Plenum, 1988, pp. 427-441.

[7] C. J. Frosch and L. Derick, "Surface protection and selective masking during diffusion in silicon," in Proc. Electrochem. Soc., 1957, p. 547.

[8] D. Khang, "Silicon—silicon dioxide surface device (technical memorandum of Bell Laboratories issued on January 16, 1961)," in Semiconductor Devices: Pioneering Papers, S. M. Sze, Ed, Singapore: World Scientific, 1991, pp. 583-596.
[9] E. Rosenbaum, J. C. King, and C. Hu, "Accelerated testing of $\mathrm{SiO}_{2}$ reliability," IEEE Trans. Electron Devices, vol. 43, pp. 70-80, 1996.

[10] G. Groeseneken, R. Degraeve, J. DeBlauwe, P. Roussel, M. Depas, and H. E. Maes, "On the breakdown statistics and mechanisms in ultra-thin oxides and nitrided oxides," in Silicon Nitride and Silicon Dioxide Thin Films, M. J. Deen, W. D. Brown, K. B. Sundaram, and S. I. Raider, Eds. Pennington, NJ: Electrochemical Soc., 1997, vol. 97-10, pp. 3-19.

[11] R. Degraeve, "Tutorial on oxide reliability," presented at the Int. Reliability Physics Symp., 1997.

[12] A. Martin, P. O'Sullivan, and A. Mathewson, "Dielectric reliability measurement methods: A review," Microelectron. Reliab., vol. 38, pp. 37-72, 1998.

[13] H. E. Maes, G. Groeseneken, R. Degraeve, J. DeBlauwe, and G. Van den bosch, "Assessment of oxide reliability and hot carrier degradation in CMOS technology," Microelectron. Eng., vol. 40, pp. 147-166, 1998.

[14] G. Ghibaudo, P. Riess, S. Bruyere, B. DeSalvo, C. Jahan, A. Scarpa, G. Pananakakis, and E. Vincent, "Emerging oxide degradation mechanisms: Stress induced leakage current (SILC) and quasibreakdown (QB)," Microelectron. Eng., vol. 49, pp. 41-50, 1999.

[15] G. Ghibaudo, G. Pananakakis, R. Kies, E. Vincent, and C. Papadas, "Accelerated dielectric breakdown and wear out standard testing methods and structures for reliability evaluation of thin oxides," Microelectron. Reliab., vol. 39, pp. 597-613, 1999.

[16] G. Groeseneken, R. Degraeve, T. Nigam, G. Van den bosch, and H. E. Maes, "Hot carrier degradation and time-dependent dielectric breakdown in oxides," Microelectron. Eng., vol. 49, pp. 27-40, 1999.

[17] R. Degraeve, B. Kaczer, and G. Groeseneken, "Degradation and breakdown in thin oxide layers: Mechanisms, models and reliability prediction," Microelectron. Reliab., vol. 39, pp. 1445-1460, 1999.

[18] E. Y. Wu, "Tutorial on ultra-thin oxide reliability for ULSI applications," presented at the Int. Integrated Reliability Workshop, Lake Tahoe, CA, 2000.

[19] D. J. DiMaria, E. Cartier, and D. Arnold, "Impact ionization, trap creation, degradation, and breakdown in silicon dioxide films on silicon," J. Appl. Phys., vol. 73, pp. 3367-3384, 1993.

[20] R. H. Fowler and L. Nordheim, "Electron emission in intense electric fields," in Proc. Royal Soc. London, vol. 119, 1928, pp. 173-181.

[21] M. Lenzlinger and E. H. Snow, "Fowler-Nordheim tunneling into thermally grown $\mathrm{SiO}_{2}$," J. Appl. Phys., vol. 40, pp. 278-283, 1969.

[22] D. J. DiMaria, E. Cartier, and D. A. Buchanan, "Anode hole injection and trapping in silicon dioxide," J. Appl. Phys., vol. 80, pp. 304-317, 1996.

[23] D. J. DiMaria and J. W. Stasiak, "Trap creation in silicon dioxide produced by hot electrons," J. Appl. Phys., vol. 65, pp. 2342-2356, 1989.

[24] D. J. DiMaria and J. H. Stathis, "Explanation for the oxide thickness dependence of breakdown characteristics of metal-oxide-semiconductor structures," Appl. Phys. Lett., vol. 70, pp. 2708-2710, 1997.

[25] D. J. DiMaria, "Electron energy dependence of metal-oxide semiconductor degradation," Appl. Phys. Lett., vol. 75, pp. 2427-2428, 1999.

[26] D. L. Griscom, "Hydrogen model for radiation-induced interface states in $\mathrm{SiO}_{2}$-on-Si structures: A review of the evidence," J. Electron. Mater. vol. 21, pp. 763-767, 1982

[27] C.-T. Sah, J. Y.-C. Sun, and J. J.-T. Tzou, "Deactivation of the boron acceptor in silicon by hydrogen," Appl. Phys. Lett., vol. 43, pp. 204-206, 1983.

[28] R. Gale, F. J. Feigl, C. W. Magee, and D. R. Young, "Hydrogen migration under avalanche injection of electrons in Si metal-oxide semiconductor capacitors," J. Appl. Phys., vol. 54, pp. 6938-6942, 1983.

[29] D. A. Buchanan, A. D. Marwick, D. J. DiMaria, and L. Dori, "Hotelectron induced hydrogen redistribution in $\mathrm{SiO}_{2}$," in The Physics and Chemistry of $\mathrm{SiO}_{2}$ and the $\mathrm{Si}_{-} \mathrm{SiO}_{2}$ Interface-2, C. R. Helms and B. E. Deal, Eds. New York, NY: Plenum, 1993, pp. 481-488.

[30] I. P. Lisovskii, V. G. Litovchenko, G. P. Romanova, P. I. Didenko, and E. G. Schmidt, "Proton release and defect creation in MOS structures due to high electric fields," Phys. Stat. Sol. (A), vol. 142, pp. 107-116, 1994.

[31] R. Degraeve, G. Groeseneken, R. Bellens, M. Depas, and H. E. Maes, "A consistent model for the thickness dependence of intrinsic breakdown in ultra-thin oxides," in Dig. 1995 Int. Electron Devices Meeting, 1995, p. 866.

[32] R. Degraeve, G. Groeseneken, R. Bellens, J. L. Ogier, M. Depas, P. J. Roussel, and H. E. Maes, "New insights in the relation between electron trap generation and the statistical properties of oxide breakdown," IEEE Trans. Electron Devices, vol. 45, pp. 904-911, 1998.

[33] J. H. Stathis, "Percolation models for gate oxide breakdown," J. Appl. Phys., vol. 86, pp. 5757-5766, 1999. 
[34] P. E. Nicollian, W. R. Hunter, and J. C. Hu, "Experimental evidence for voltage driven breakdown models in ultrathin gate oxides," in Proc. 2000 Int. Reliability Physics Symp., 2000, pp. 7-15.

[35] C.-T. Sah, "Origin of interface states and oxide charges generated by ionizing radiation," IEEE Trans. Nucl. Sci., vol. NS-23, pp. 1563-1568, 1976.

[36] A. G. Revesz, "The role of hydrogen in $\mathrm{SiO}_{2}$ films on silicon," J. Electrochem. Soc., vol. 126, pp. 122-130, 1979.

[37] L. do Thanh and P. Balk, "Elimination and generation of $\mathrm{Si}_{-} \mathrm{SiO}_{2}$ interface traps by low temperature hydrogen annealing," J. Electrochem. Soc., vol. 135, pp. 1797-1801, 1988

[38] E. Cartier, J. H. Stathis, and D. A. Buchanan, "Passivation and depassivation of silicon dangling bonds at the $\mathrm{Si} / \mathrm{SiO}_{2}$ interface by atomic hydrogen," Appl. Phys. Lett., vol. 63, pp. 1510-1512, 1993.

[39] J. H. Stathis and E. Cartier, "Atomic hydrogen reactions with $P_{b}$ centers at the (100) $\mathrm{Si} / \mathrm{SiO}_{2}$ interface," Phys. Rev. Lett., vol. 72, pp. 2745-2748, 1994.

[40] R. E. Stahlbush and E. Cartier, "Interface defect formation in MOSFET's by atomic hydrogen exposure," IEEE Trans. Nucl. Sci., vol. 41, pp. 1844-1853, 1994

[41] E. Cartier, D. A. Buchanan, J. H. Stathis, and D. J. DiMaria, "Atomic hydrogen-induced degradation of thin $\mathrm{SiO}_{2}$ gate dielectrics," J. NonCryst. Solids, vol. 187, pp. 244-247, 1995.

[42] K. G. Druijf, J. M. M. de Nijs, E. v.d. Drift, V. V. Afanas'ev, E. H. A. Granneman, and P. Balk, "The microscopic nature of donor-type $\mathrm{Si}_{-} \mathrm{SiO}_{2}$ interface states," J. Non-Cryst. Solids, vol. 187, pp. 206-210, 1995.

[43] E. Cartier and J. H. Stathis, "Atomic hydrogen-induced degradation of the $\mathrm{Si} / \mathrm{SiO}_{2}$ structure," Microelectron. Eng., vol. 28, pp. 3-10, 1995.

[44] D. J. DiMaria and E. Cartier, "Mechanism for stress-induced leakage currents in thin silicon dioxide films," J. Appl. Phys., vol. 78, pp. 3883-3894, 1995.

[45] E. Cartier and J. H. Stathis, "Hot-electron induced passivation of silicon dangling bonds at the $\mathrm{Si}(111) / \mathrm{SiO}_{2}$ interface," Appl. Phys. Lett., vol. 69, pp. 103-105, 1996

[46] R. E. Stahlbush, "Slow and fast state formation caused by hydrogen," in The Physics and Chemistry of $\mathrm{SiO}_{2}$ and the $\mathrm{Si}-\mathrm{SiO}_{2}$ Interface- $-3, \mathrm{H}$. Z. Massoud, C. R. Helms, and E. H. Poindexter, Eds. Pennington, NJ: Electrochemical Soc., 1996, vol. 96-1, pp. 525-537.

[47] A. Szekeres and S. Alexandra, "Low-temperature treatment of $\mathrm{Si} / \mathrm{SiO}_{2}$ structures in an RF hydrogen plasma," Vacuum, vol. 47, pp. 1483-1486, 1996.

[48] J. H. Stathis and E. Cartier, "The role of atomic hydrogen in degradation and breakdown of $\mathrm{SiO}_{2}$ films," in Extended Abstracts of the 1996 Int. Conf. on Solid State Devices and Materials, 1996, pp. 791-793.

[49] J. M. M. de Nijs, K. G. Druijf, and V. V. Afanas'ev, "Hydrogen-induced donor states in the MOS system: Hole traps, slow states and interface states," in Fundamental Aspects of Ultrathin Dielectrics on Si-Based Devices, NATO Science Series, 3/47 ed, E. Garfunkel, E. Gusev, and A. Vul', Eds. Dortrecht, The Netherlands: Kluwer, 1998, pp. 425-430.

[50] J. H. Stathis, "Electrical defects at the $\mathrm{SiO}_{2} / \mathrm{Si}$ interface studied by EPR," in Fundamental Aspects of Ultrathin Dielectrics on Si-Based Devices, NATO Science Series, 3/47 ed, E. Garfunkel, E. Gusev, and A. Vul', Eds. Dortrecht, The Netherlands: Kluwer, 1998, pp. 325-333.

[51] E. H. Poindexter, C. F. Young, and G. J. Gerardi, "Hydrogenous species and charge defects in the $\mathrm{Si}-\mathrm{SiO}_{2}$ system," in Fundamental Aspects of Ultrathin Dielectrics on Si-Based Devices, NATO Science Series, 3/47 ed, E. Garfunkel, E. Gusev, and A. Vul', Eds. Dortrecht, The Netherlands: Kluwer, 1998, pp. 397-410.

[52] T.-C. Shen, C. Wang, G. C. Abeln, J. R. Tucker, J. W. Lyding, P. Avouris, and R. E. Walkup, "Atomic-scale desorption through electronic and vibrational excitation mechanisms," Science, vol. 268, pp. 1590-1592, 1995.

[53] J. Wu, E. Rosenbaum, B. MacDonald, E. Li, J. Tao, B. Tracy, and P. Fang, "Anode hole injection versus hydrogen release: The mechanism for gate oxide breakdown," in Proc. 2000 Int. Reliability Physics Symp., 2000 , pp. 27-32.

[54] K. Hess, I. C. Kizilyalli, and J. W. Lyding, "Giant isotope effect in hot electron degradation of metal oxide silicon devices," IEEE Trans. Electron Devices, vol. 45, pp. 406-416, 1998.

[55] Y. Mitani, H. Satake, H. Ito, and A. Toriumi, "A study of the effect of deuterium on stress induced leakage," Jpn. J. Appl. Phys. 2, vol. 39, pp. 564-566, 2000.

[56] _ _Highly reliable gate oxide under Fowler-Nordheim electron injection by deuterium pyrogenic oxidation and deuterated poly-Si deposition," in Dig. 2000 Int. Electron Devices Meeting, 2000, pp. 343-346.
[57] D. J. Dumin and J. R. Maddux, "Correlation of stress-induced leakage current in thin oxides with trap generation inside the oxides," IEEE Trans. Electron Devices, vol. 40, pp. 986-993, 1993.

[58] M. Kimura and H. Koyama, "Stress-induced low-level leakage mechanism of ultrathin silicon dioxide films caused by neutral oxide trap generation," in Proc. 1994 Int. Reliability Physics Symp., 1994, pp. $167-172$.

[59] H. Kim and H. Hwang, "High-quality ultrathin gate oxide prepared by oxidation in $\mathrm{D}_{2} \mathrm{O}$," Appl. Phys. Lett., vol. 74, pp. 709-710, 1999.

[60] I. C. Chen, S. E. Holland, K. K. Young, C. Chang, and C. Hu, "Substrate hole current and oxide breakdown," Appl. Phys. Lett., vol. 49, pp. 669-671, 1986

[61] C. Schuegraf and C. Hu, "Metal-oxide semiconductor field-effect-transistor substrate current during Fowler-Nordheim tunneling stress and silicon dioxide reliability," J. Appl. Phys., vol. 76, pp. 3695-3700, 1994.

[62] M. V. Fischetti, "Model for the generation of positive charge at the $\mathrm{Si}-\mathrm{SiO}_{2}$ interface based on hot-hole injection from the interface," Phys. Rev. B., vol. 31, pp. 2099-2113, 1985.

[63] J.-H. Kim, J. J. Sanchez, T. A. DeMassa, M. T. Quddus, D. Smith, F. Shaapur, K. Weiss, and C. H. Liu, "Surface plasmons and breakdown in thin silicon dioxide films on silicon," J. Appl. Phys., vol. 84, pp. $1430-1438,1998$.

[64] X. Gao and S. S. Yee, "Threshold energies of high-field-induced hole currents and positive charges in $\mathrm{SiO}_{2}$ layers of metal-oxide-semiconductor structures," J. Appl. Phys., vol. 76, pp. 5795-5799, 1994.

[65] H. Satake and A. Toriumi, "Temperature dependent hole fluence to breakdown in thin gate oxides under Fowler-Nordheim electron tunneling," Appl. Phys. Lett., vol. 66, pp. 3516-3517, 1995.

[66] J. C. Lee, I. C. Chen, and C. Hu, "Modeling and characterization of gate oxide reliability," IEEE Trans. Electron Devices, vol. 35, pp. 2268-2278, 1988

[67] M. A. Alam, J. D. Bude, and A. Ghetti, "Field acceleration for oxide breakdown-Can an accurate anode hole injection model resolve the E vs. 1/E controversy?," in Proc. 2000 Int. Reliability Physics Symp., 2000, pp. $21-26$.

[68] J. D. Bude, B. E. Weir, and P. J. Silverman, "Explanation of stressinduced damage in thin oxides," in Dig. 1998 Int. Electron Devices Meeting, 1998, pp. 179-182.

[69] M. A. Alam, B. E. Weir, P. J. Silverman, J. D. Bude, A. Ghetti, Y. Ma, M. M. Brown, D. Hwang, and A. Hamad, "Physics and prospects of sub-2nm oxides," in The Physics and Chemistry of $\mathrm{SiO}_{2}$ and the $\mathrm{Si}_{-} \mathrm{SiO}_{2}$ Interface - 4, H. Z. Massoud, I. J. R. Baumvol, M. Hirose, and E. H. Poindexter, Eds. Pennington, NJ: Electrochem. Soc., 2000, vol. 2000-2, pp. 365-376.

[70] Y. Lu and C.-T. Sah, "Two pathways of positive oxide-charge buildup during electron tunneling into silicon dioxide film," J. Appl. Phys., vol. 76, pp. 4724-4727, 1994.

[71] D. J. DiMaria, "Defect generation in ultrathin silicon dioxide films produced by anode hole injection," Appl. Phys. Lett., vol. 77, pp. $2716-2718,2000$.

[72] D. J. DiMaria and J. H. Stathis, "Anode hole injection, defect generation, and breakdown in ultrathin silicon dioxide films," J. Appl. Phys., vol. 89, pp. 5015-5024, 2001.

[73] T. Tomita, H. Utsunomiya, Y. Kamakura, and K. Taniguchi, "Hot hole induced breakdown of thin silicon dioxide films," Appl. Phys. Lett., vol. 71, pp. 3664-3666, 1997.

[74] E. M. Vogel and J. S. Suehle, private communication unpublished

[75] Y. Shi, T. P. Ma, S. Prasad, and S. Dhanda, "Polarity-dependent tunneling current and oxide breakdown in dual-gate CMOSFETs," IEEE Electron Device Lett., vol. 19, pp. 391-393, 1998.

[76] M. Rasras, I. De Wolf, G. Groeseneken, B. Kaczer, R. Degraeve, and H. E. Maes, "Photo-carrier generation as the origin of Fowler-Nordheiminduced substrate hole current in thin oxides," in Dig. 1999 Int. Electron Devices Meeting, 1999, pp. 465-468.

[77] J. H. Stathis and D. J. DiMaria, "Defect generation and reliability of ultra-thin $\mathrm{SiO}_{2}$ at low voltage," in The Physics and Chemistry of $\mathrm{SiO}_{2}$ and the $\mathrm{Si}_{-} \mathrm{SiO}_{2}$ Interface - 4, H. Z. Massoud, I. J. R. Baumvol, M. Hirose, and E. H. Poindexter, Eds. Pennington, NJ: Electrochem. Soc., 2000, vol. 2000-2, pp. 33-44.

[78] J. W. McPherson and H. C. Mogul, "Underlying physics of the thermochemical E model in describing low-field time-dependent breakdown in $\mathrm{SiO}_{2}$ thin films," J. Appl. Phys., vol. 84, pp. 1513-1523, 1998.

[79] J. W. McPherson and R. B. Khamankar, "Molecular model for intrinsic time-dependent dielectric breakdown in $\mathrm{SiO}_{2}$ dielectrics and the reliability implications for hyper-thin gate oxide," Semicond. Sci. Technol., vol. 15, pp. 462-470, 2000. 
[80] G. A. Swartz, "Gate oxide integrity of NMOS transistor arrays," IEEE Trans. Electron Devices, vol. ED-33, pp. 1826-1829, 1986.

[81] M. Kimura, H. Koyama, and A. Yasuoka, "Field acceleration model for time-dependent dielectric breakdown," in Extended Abstracts of the 1996 Int. Conf. on Solid State Devices and Materials, 1996, pp. 530-532.

[82] E. Vincent, N. Revil, C. Papadas, and G. Ghibaudo, "Electric field dependence of TDDB activation energy in ultrathin oxides," Microelectron. Reliab., vol. 36, pp. 1643-1646, 1996.

[83] J. W. McPherson, R. B. Khamankar, and A. Shanware, "A complementary molecular-model (including field and current) for TDDB in $\mathrm{SiO}_{2}$ dielectrics," Microelectron. Reliab., vol. 40, pp. 1591-1597, 2000.

[84] K. P. Cheung, "A physics-based unified gate-oxide breakdown model," in Dig. 1999 Int. Electron Devices Meeting, 1999, pp. 719-722.

[85] C. Hu and Q. Lu, "A unified gate oxide reliability model," in Proc. 1999 Int. Reliability Physics Symp., 1999, pp. 47-51.

[86] K. Okada and K. Yoneda, "A consistent model for time dependent dielectric breakdown in ultrathin silicon dioxides," in Dig. 1999 Int. Electron Devices Meeting, 1999, pp. 445-447.

[87] J. S. Suehle, P. Chaparala, C. Messick, W. M. Miller, and K. C. Boyko, "Field and temperature acceleration of time-dependent dielectric breakdown in intrinsic thin $\mathrm{SiO}_{2}$," in Proc. 1994 Int. Reliability Physics Symp., 1994, pp. 120-125.

[88] D. J. Dumin, S. K. Mopuri, S. Vanchinathan, R. S. Scott, R. Subramoniam, and T. G. Lewis, "High field related thin oxide wearout and breakdown," IEEE Trans. Electron Devices, vol. 42, pp. 760-772, 1995.

[89] M. Kimura, "Field and temperature acceleration model for time-dependent dielectric breakdown," IEEE Trans. Electron Devices, vol. 46, pp 220-229, 1999.

[90] M. Kimura and H. Koyama, "Mechanism of time-dependent oxide breakdown in thermally grown $\mathrm{SiO}_{2}$ films," J. Appl. Phys., vol. 85, pp. 7671-7681, 1999.

[91] J. McPherson, V. Reddy, K. Banerjee, and H. Le, "Comparison of E and 1/E TDDB models for $\mathrm{SiO}_{2}$ under long-term/low-field test conditions," in Dig. 1998 Int. Electron Devices Meeting, 1998, pp. 171-174.

[92] K. Umeda, T. Tomita, and K. Taniguchi, "Silicon dioxide breakdown induced by SHE (substrate hot electron) injection," Electronics and Communications in Japan, pt. 2, vol. 80, pp. 11-19, 1997.

[93] E. M. Vogel, J. S. Suehle, M. D. Edelstein, B. Wang, Y. Chen, and J. B. Bernstein, "Reliability of ultrathin silicon dioxide under combined substrate hot-electron and constant voltage stress," IEEE Trans. Electron Devices, vol. 47, pp. 1183-1191, 2000.

[94] J. F. Verwey, "Nonavalanche injection of hot carriers into $\mathrm{SiO}_{2}$," J. Appl. Phys., vol. 44, pp. 2681-2687, 1973.

[95] D. J. DiMaria, "Explanation for the polarity dependence of breakdown in ultrathin silicon dioxide films," Appl. Phys. Lett., vol. 68, pp. 3004-3006, 1996.

[96] J. M. McKenna, E. Y. Wu, and S.-H. Lo, "Tunneling current characteristics and oxide breakdown in $\mathrm{p}^{+}$poly gate PFET capacitors," in Proc. 2000 Int. Reliability Physics Symp., 2000, pp. 16-20.

[97] B. Ricco, M. Y. Azbel, and M. H. Brodsky, "Novel mechanism for tunneling and breakdown in thin $\mathrm{SiO}_{2}$ films," Phys. Rev. Lett., vol. 51, pp. $1795-1798,1983$.

[98] Y. Nissan-Cohen, J. Shappir, and D. Frohman-Bentchkowsky, "Trap generation and occupation dynamics in $\mathrm{SiO}_{2}$ under charge injection stress," J. Appl. Phys., vol. 60, pp. 2024-2035, 1986.

[99] D. Qian and D. J. Dumin, "The field, time and fluence dependencies of trap generation in silicon oxides between 5 and $13.5 \mathrm{~nm}$ thick," Semicond. Sci. Technol., vol. 15, pp. 854-861, 2000

[100] M. A. Alam, J. D. Bude, B. Weir, P. Silverman, A. Ghetti, D. Monroe, K. P. Cheung, and S. Moccio, "An anode hole injection percolation model for oxide breakdown-The "doom's day" scenario revisited," in Dig. 1999 Int. Electron Devices Meeting, 1999, pp. 715-718.

[101] P. P. Apte and K. C. Saraswat, "Correlation of trap generation to charge-to-breakdown $\left(Q_{\mathrm{bd}}\right)$ : A physical-damage model of dielectric breakdown," IEEE Trans. Electron Devices, vol. 41, pp. 1595-1602, 1994.

[102] D. J. Dumin, J. R. Maddux, R. S. Scott, and R. Subramoniam, "A model relating wearout to breakdown in thin oxides," IEEE Trans. Electron Devices, vol. 41, pp. 1570-1580, 1994.

[103] D. J. DiMaria, "Defect production, degradation, and breakdown of silicon dioxide films," Solid-State Electron., vol. 41, pp. 957-965, 1997.

[104] J. H. Stathis and D. J. DiMaria, "Reliability projection for ultra-thin oxides at low voltage," in Dig. 1998 Int. Electron Devices Meeting, 1998, pp. $167-170$.
[105] R. Rodríguez, E. Miranda, R. Pau, J. Suñé, M. Nafría, and X. Aymerich, "Monitoring the degradation that causes the breakdown of ultrathin $(<5 \mathrm{~nm}) \mathrm{SiO}_{2}$ gate oxides," IEEE Electron Device Lett., vol. 21, pp. $251-253,2000$

[106] J. Suñé, I. Placencia, N. Barniol, E. Farrés, F. Martín, and X. Aymerich, "On the breakdown statistics of very thin $\mathrm{SiO}_{2}$ films," Thin Solid Films, vol. 185 , pp. 347-362, 1990 .

[107] S. Lombardo, A. La Magna, C. Spinella, C. Gerardi, and F. Crupi, "Degradation and hard breakdown transient of thin gate oxides in metal- $\mathrm{SiO}_{2}-\mathrm{Si}$ capacitors: Dependence on oxide thickness," J. Appl. Phys., vol. 86, pp. 6382-6391, 1999.

[108] M. A. Alam, B. E. Weir, P. J. Silverman, Y. Ma, and D. Hwang, "The statistical distribution of percolation resistance as a probe into the mechanics of ultra-thin oxide breakdown," in Dig. 2000 Int. Electron Devices Meeting, 2000, pp. 529-532.

[109] K. Okada, H. Kubo, A. Ishinaga, and K. Yoneda, "A new prediction method for oxide lifetime and its application to study dielectric breakdown mechanism," in Dig. 1998 Symp. on VLSI Technology, 1998, pp. 158-159.

[110] T. Nigam, R. Degraeve, G. Groeseneken, M. M. Heyns, and H. E. Maes, "A fast and simple methodology for lifetime projection of ultra-thin oxides," in Proc. 1999 Int. Reliability Physics Symp., 1999, pp. 381-388.

[111] B. Kaczer, R. Degraeve, N. Pangon, and G. Groeseneken, "The influence of elevated temperature on degradation and lifetime prediction of thin silicon-dioxide films," IEEE Trans. Electron Devices, vol. 47, pp. 1514-1521, 2000

[112] R. Degraeve, B. Kaczer, and G. Groeseneken, "Reliability: A possible showstopper for oxide thickness scaling?," Semicond. Sci. Technol., vol. 15 , pp. 436-444, 2000

[113] A. Ghetti, J. D. Bude, and G. Weber, " $T_{\mathrm{BD}}$ prediction from measurements at low field and room temperature using a new estimator," in Dig. 2000 Symp. on VLSI Technology, 2000, pp. 218-219.

[114] Y. Kamakura, H. Utsunomiya, T. Tomita, K. Umeda, and K. Taniguchi, "Investigations of hot-carrier-induced breakdown of thin oxides," in Dig. 1997 Int. Electron Devices Meeting, 1997, pp. 81-84.

[115] K. Umeda and K. Taniguchi, "Hot-electron-induced quasibreakdown of thin gate oxides," J. Appl. Phys., vol. 82, pp. 297-302, 1997.

[116] E. Vincent, S. Bruyere, C. Papadas, and P. Mortini, "Dielectric reliability in deep submicron technologies: From thin to ultrathin oxides," Microelectron. Reliab., vol. 37, pp. 1499-1506, 1997.

[117] E. Vincent, C. Papadas, and G. Ghibaudo, "Temperature dependence of charge build up mechanisms and breakdown phenomena in thin oxides under Fowler Nordheim injection," Solid-State Electron., vol. 41, p. 1001, 1997.

[118] M. Houssa, P. W. Mertens, and M. M. Heyns, "Relation between stressinduced leakage current and time-dependent dielectric breakdown in ultra-thin gate oxides," Semicond. Sci. Technol., vol. 14, pp. 892-896, 1999.

[119] D. J. DiMaria and J. H. Stathis, "Ultimate limit for defect generation in ultra-thin silicon dioxide," Appl. Phys. Lett., vol. 71, pp. 3230-3232, 1997.

[120] K. P. Cheung, C. T. Liu, C.-P. Chang, J. I. Colonell, W. Y.-C. Lai, R. Liu, J. F. Miner, C. S. Pai, H. Vaidya, J. T. Clemens, and E. Hasegawa, "Field dependent critical trap density for thin gate oxide breakdown," in Proc. 1999 Int. Reliability Physics Symp., 1999, pp. 52-56.

[121] K. Okada, "Analysis of the relationship between defect site generation and dielectric breakdown utilizing A-mode stress induced leakage current," IEEE Trans. Electron Devices, vol. 47, pp. 1225-1230, 2000.

[122] A. Teramoto, H. Umeda, K. Azamawari, K. Kobayashi, K. Shiga, J. Komori, Y. Ohno, and H. Miyoshi, "Study of oxide breakdown under very low electric field," in Proc. 1999 Int. Reliability Physics Symp., 1999, pp. 66-71.

[123] J. H. Stathis, A. Vayshenker, P. R. Varekamp, E. Y. Wu, C. Montrose, J. McKenna, D. J. DiMaria, L.-K. Han, E. Cartier, R. A. Wachnik, and B P. Linder, "Breakdown measurements of ultra-thin $\mathrm{SiO}_{2}$ at low voltage," in Dig. 2000 Symp. on VLSI Technology, 2000, pp. 94-95.

[124] L. Niemeyer, L. Pietronero, and H. J. Wiesmann, "Fractal dimension of dielectric breakdown," Phys. Rev. Lett., vol. 52, pp. 1033-1036, 1984.

[125] T. Tanamoto and A. Toriumi, "A percolation approach to dielectric breakdown statistics," Jpn. J. Appl. Phys., vol. 36, pp. 1439-1442, 1997.

[126] D. J. DiMaria, "Defect generation under substrate-hot-electron injection into ultrathin silicon dioxide layers," J. Appl. Phys., vol. 86, pp. 2100-2109, 1999.

[127] — - "Defect generation in field-effect transistors under channel-hotelectron stress," J. Appl. Phys., vol. 87, pp. 8707-8715, 2000. 
[128] M. Depas, B. Vermeire, P. W. Mertens, M. Meuris, and M. M. Heyns, "Wear-out of ultra-thin gate oxides during high-field electron tunneling," Semicond. Sci. Technol., vol. 10, pp. 753-758, 1995.

[129] M. Depas, B. Vermeire, and M. M. Heyns, "Breakdown and defect generation in ultrathin gate oxide," J. Appl. Phys., vol. 80, pp. 382-387, 1996.

[130] T. Ghani, S. Ahmed, P. Aminzadeh, J. Bielefeld, P. Charvat, C. Chu, M. Harper, P. Jacob, C. Jan, J. Kavalieros, C. Kenyon, R. Nagisetty, P. Packan, J. Sebastian, M. Taylor, J. Tsai, S. Tyagi, S. Yang, and M. Bohr, "100 nm gate length high performance/low power CMOS transistor structure," in Dig. 1999 Int. Electron Devices Meeting, 1999, pp. $415-418$.

[131] E. J. Gumbel, Statistical Theory of Extreme Values and Some Practical Applicatons. Washington, D.C., U.S.: Government Printing Office, 1954, vol. 33. National Bureau of Standards Applied Mathematics Series.

[132] D. R. Wolters, "Breakdown and wearout phenomena in $\mathrm{SiO}_{2}$," in Insulating Films on Semiconductors, M. Schulz and G. Pensl, Eds. Berlin, Germany: Springer-Verlag, 1981, pp. 180-194.

[133] W. G. Kleppmann, "Reliability predictions for integrated circuits from test structure data," Quality and Reliability Eng. Int., vol. 5, pp. 155-163, 1989.

[134] W. Nelson, Accelerated Testing. New York: Wiley, 1990

[135] E. Y. Wu, J. H. Stathis, and L.-K. Han, "Ultra-thin oxide reliability for ULSI applications," Semicond. Sci. Technol., vol. 15, pp. 425-435, 2000.

[136] E. Y. Wu, D. L. Harmon, and L.-K. Han, "Interrelationship of voltage and temperature dependence of oxide breakdown for ultrathin oxides," IEEE Electron Device Lett., vol. 21, pp. 362-364, 2000.

[137] E. Y. Wu, E. Nowak, L.-K. Han, R. Dufresne, and W. W. Abadeer, "Nonlinear characteristics of Weibull breakdown distributions and its impact on reliability projection for ultra-thin oxides," in Dig. 1999 Int. Electron Devices Meeting, 1999, pp. 441-444.

[138] B. E. Weir, P. J. Silverman, M. A. Alam, F. Baumann, D. Monroe, A. Ghetti, J. D. Bude, G. L. Timp, A. Hamad, T. M. Oberdick, N. X. Zhao, Y. Ma, M. M. Brown, D. Hwang, T. Sorsch, and J. Madic, "Gate oxides in $50 \mathrm{~nm}$ devices: Thickness uniformity improves projected reliability," in Dig. 1999 Int. Electron Devices Meeting, 1999, pp. 437-440.

[139] J. Jacquelin, "Inference of sampling on Weibull parameter estimation," IEEE Trans. Dielect. Elect. Insulation, vol. 3, pp. 809-816, 1996.

[140] E. Y. Wu, W. W. Abadeer, L.-K. Han, S.-H. Lo, and G. Hueckel, "Challenges for accurate reliability projections in the ultra-thin oxide regime," in Proc. 1999 Int. Reliability Physics Symp., 1999, pp. 57-65.

[141] D. J. DiMaria and J. H. Stathis, "Non-arrhenius temperature dependence of reliability in ultrathin silicon dioxide films," Appl. Phys. Lett., vol. 74, pp. 1752-1754, 1999.

[142] B. Kaczer, R. Degraeve, N. Pangon, T. Nigam, and G. Groeseneken, "Investigation of temperature acceleration of thin oxide time-to-breakdown," Microelectron. Eng., vol. 48, pp. 47-50, 1999.

[143] International Technology Roadmap for Semiconductors (ITRS) (2000). [Online]. Available: http://public.itrs.net/

[144] L.-K. Han, S. Crowder, M. Hargrove, E. Wu, S.-H. Lo, F. Guarin, E. Crabbé, and L. Su, "Electrical characteristics and reliability of sub-3 nm gate oxides grown on nitrogen implanted silicon substrates," in Dig. 1997 Int. Electron Devices Meeting, 1997, pp. 643-646.

[145] P. E. Nicollian, G. C. Baldwin, K. N. Eason, D. T. Grider, S. V. Hattangady, J. C. Hu, W. R. Hunter, M. Rodder, and A. L. P. Rotondaro, "Extending the reliability scaling limit of $\mathrm{SiO}_{2}$ through plasma nitridation," in Dig. 2000 Int. Electron Devices Meeting, 2000, pp. 545-548.

[146] B. E. Weir, P. J. Silverman, D. Monroe, K. S. Krisch, M. A. Alam, G. B. Alers, T. W. Sorsch, G. L. Timp, F. Baumann, C. T. Liu, Y. Ma, and D. Huang, "Ultra-thin gate dielectrics: They break down, but do they fail?," in Dig. 1997 Int. Electron Devices Meeting, 1997, pp. 73-76.

[147] K. Okada, H. Kubo, A. Ishinaga, and K. Yoneda, "A concept of gate oxide lifetime limited by 'B-mode' stress induced leakage currents in direct tunneling regime," in Dig. 1999 Symp. on VLSI Technology, 1999, pp. 57-58.

[148] K. Okada, "The gate oxide lifetime limited by 'B-mode' stress induced leakage current and the scaling limit of silicon dioxides in the direct tunneling regime," Semicond. Sci. Technol., vol. 15, pp. 478-484, 2000.

[149] M. Houssa, T. Nigam, P. W. Mertens, and M. M. Heyns, "Soft breakdown in ultrathin gate oxides: Correlation with the percolation theory of nonlinear conductors," Appl. Phys. Lett., vol. 73, pp. 514-516, 1998.

[150] _ " "Model for the current-voltage characteristics of ultrathin gate oxides after soft breakdown," J. Appl. Phys., vol. 84, pp. 4351-4355, 1998.

[151] J. Suñé, E. Miranda, M. Nafría, and X. Aymerich, "Point contact conduction at the oxide breakdown of MOS devices," in Dig. 1998 Int. Electron Devices Meeting, 1998, pp. 191-194.
[152] N. Vandewalle, M. Ausloos, M. Houssa, P. W. Mertens, and M. M. Heyns, "Non-gaussian behavior and anticorrelations in ultrathin gate oxides after soft breakdown," Appl. Phys. Lett., vol. 74, pp. 1579-1581, 1999.

[153] E. Miranda, J. Suñé, R. Rodríguez, M. Nafría, F. Martín, and X. Aymerich, "Soft breakdown in ultrathin $\mathrm{SiO}_{2}$ layers: The conduction problem from a new point of view," Jpn. J. Appl. Phys., vol. 38, pp. 2223-2226, 1999.

[154] J. Suñé, E. Miranda, M. Nafría, and X. Aymerich, "Modeling the breakdown spots in silicon dioxide films as point contacts," Appl. Phys. Lett., vol. 75, pp. 959-961, 1999.

[155] E. Miranda, J. Suñé, R. Rodríguez, M. Nafría, X. Aymerich, L. Fonseca, and F. Campabadal, "Soft breakdown in ultrathin (3-5 nm) gate dielectrics," IEEE Trans. Electron Devices, vol. 47, pp. 82-89, 2000

[156] E. Miranda and J. Suñe, "Modeling the conduction characteristics of broken down gate oxides in MOS structures," Microelectron. Reliab., vol. 40, pp. 1599-1603, 2000.

[157] J. Suñé, M. Nafría, X. Oriols, R. Rodríguez, and X. Aymerich, "Failure physics of ultra-thin $\mathrm{SiO}_{2}$ gate oxides near their scaling limit," Semicond. Sci. Technol., vol. 15, pp. 445-454, 2000.

[158] J. Suñé and E. Miranda, "Soft and hard breakdown of ultrathin $\mathrm{SiO}_{2}$ gate oxides," in The Physics and Chemistry of $\mathrm{SiO}_{2}$ and the $\mathrm{Si}_{-} \mathrm{SiO}_{2}$ Interface-4, H. Z. Massoud, I. J. R. Baumvol, M. Hirose, and E. H. Poindexter, Eds. Pennington, NJ: Electrochem. Soc., 2000, vol. 2000-2, pp. 333-344.

[159] _ "Post soft breakdown condution in $\mathrm{SiO}_{2}$ gate oxides," in Dig. 2000 Int. Electron Devices Meeting, 2000, pp. 533-536.

[160] K. Okada, S. Kawasaki, and Y. Hirofuji, "New experimental findings on stress induced leakage current of ultra thin silicon dioxides," in Extended Abstracts 1994 Int. Conf. Solid State Devices and Materials, 1994, pp. 565-567.

[161] S.-H. Lee, B.-J. Cho, J.-C. Kim, and S.-H. Choi, "Quasibreakdown of ultrathin gate oxide under high field stress," in Dig. 1994 Int. Electron Devices Meeting, 1994, pp. 605-607.

[162] M. Depas, T. Nigam, and M. M. Heyns, "Soft breakdown of ultra-thin gate oxide layers," IEEE Trans. Electron Devices, vol. 43, pp. 2499-1504, 1996.

[163] J. C. Jackson, T. Robinson, O. Oralkan, D. J. Dumin, and G. A. Brown, "Nonuniqueness of time-dependent-dielectric-breakdown distributions," Appl. Phys. Lett., vol. 71, pp. 3682-3684, 1997.

[164] —_ "Differentiation between electric breakdowns and dielectric breakdown in thin silicon oxides," J. Electrochem. Soc., vol. 45, pp. 1033-1038, 1997.

[165] S. Lombardo, F. Crupi, A. La Magna, C. Spinella, A. Terrasi, A. La Mantia, and B. Neri, "Electrical and thermal transient during dielectric breakdown of thin oxides in metal- $\mathrm{SiO}_{2}$ capacitors," J. Appl. Phys., vol. 84, pp. 472-479, 1998.

[166] S. Lombardo, F. Crupi, C. Spinella, and B. Neri, "Transients during prebreakdown and hard breakdown of thin gate oxides in metal- $\mathrm{SiO}_{2}-\mathrm{Si}$ capacitors," Mater. Sci. Semicond. Processing, vol. 2, pp. 359-367, 1999.

[167] S. Lombardo, A. La Magna, C. Gerardi, M. Alessandri, and F. Crupi, "Soft breakdown of gate oxides in metal- $\mathrm{SiO}_{2}-\mathrm{Si}$ capacitors under stress with hot electrons," Appl. Phys. Lett., vol. 75, pp. 1161-1163, 1999.

[168] P. Blöchl and J. H. Stathis, "Aspects of defects in silica related to dielectric breakdown of gate oxides in MOSFET's," Physica B, vol. 273-274, pp. 1022-1026, 1999.

[169] M. A. Alam, B. Weir, J. Bude, P. Silverman, and D. Monroe, "Explanation of soft and hard breakdown and its consequences for area scaling," in Dig. 1999 Int. Electron Devices Meeting, 1999, pp. 449-452.

[170] H. Satake and A. Toriumi, "Dielectric breakdown mechanism of thin- $\mathrm{SiO}_{2}$ studied by the post-breakdown resistance statistics," IEEE Trans. Electron Devices, vol. 47, pp. 741-745, 2000

[171] — " $\mathrm{SiO}_{2}$ dielectric breakdown mechanism studied by the post-breakdown resistance statistics," Semicond. Sci. Technol., vol. 15, pp. 471-477, 2000

[172] B. P. Linder, J. H. Stathis, R. A. Wachnik, E. Wu, S. A. Cohen, A. Ray, and A. Vayshenker, "Gate oxide breakdown under current limited constant voltage stress," in Dig. 2000 Symp. on VLSI Technology, 2000, pp. $214-215$.

[173] A. Toriumi and H. Satake, "The boundary between hard- and soft-breakdown in ultra-thin silicon dioxide films," in Structure and Electronic Properties of Ultrathin Dielectric Films on Silicon and Related Structures, Materials Research Society Symposium Proceedings, D. A. Buchanan, A. H. Edwards, H. J. von Bardeleben, and T. Hattori, Eds. Warrendale, PA: Materials Research Soc., 2000, vol. 592, pp. 323-329. 
[174] A. Toriumi, S. Takagi, and H. Satake, "Study of soft breakdown in thin $\mathrm{SiO}_{2}$ films by carrier-separation technique and breakdown transient modulation," in The Physics and Chemistry of $\mathrm{SiO}_{2}$ and the $\mathrm{Si}-\mathrm{SiO}_{2}$ Interface - 4, H. Z. Massoud, I. J. R. Baumvol, M. Hirose, and E. H. Poindexter, Eds. Pennington, NJ: Electrochem. Soc., 2000, vol. 2000-2, pp. 399-407.

[175] K. Okada, "Extended time dependent dielectric breakdown model based on anomalous gate area dependence of lifetime in ultra thin silicon dioxides," Jpn. J. Appl. Phys., vol. 36, pp. 1443-1447, 1997.

[176] J. Suñé, G. Mura, and E. Miranda, "Are soft breakdown and hard breakdown of ultrathin gate oxides actually different failure mechanisms?," IEEE Electron Device Lett., vol. 21, pp. 167-169, 2000.

[177] E. Wu, E. Nowak, J. Aitken, W. Abadeer, L. K. Han, and S. H. Lo, "Structural dependence of dielectric breakdown in ultra-thin gate oxides and its relationship to soft breakdown modes and device failure," in Dig. 1998 Int. Electron Devices Meeting, 1998, pp. 187-190.

[178] T. Pompl, H. Wurzer, M. Kerber, and I. Eisele, "Investigation of ultra-thin gate oxide reliability behavior by separate characterization of soft breakdown and hard breakdown," in Proc. 2000 Int. Reliability Physics Symp., 2000, pp. 40-47.

[179] J. S. Suehle, E. M. Vogel, B. Wang, and J. B. Bernstein, "Temperature dependence of soft breakdown and wear-out in sub-3 nm SiO 2 films," in Proc. 2000 Int. Reliability Physics Symp., 2000, pp. 33-39.

[180] M. Rasras, I. De Wolf, G. Groeseneken, R. Degraeve, and H. E. Maes, "Substrate hole current after oxide breakdown," in Dig. 2000 Int. Electron Devices Meeting, 2000, pp. 537-540.

[181] S. Bruyere, E. Vincent, and G. Ghibaudo, "Quasibreakdown in ultra-thin $\mathrm{SiO}_{2}$ films: Occurence characterization and reliability assessment methodology," in Proc. 2000 Int. Reliability Physics Symp. 2000, pp. 48-54.

[182] B. E. Weir, P. J. Silverman, G. B. Alers, G. B. Monroe, M. A. Alam, T. W. Sorsch, M. L. Green, G. L. Timp, Y. Ma, Y. Frei, C. T. Liu, and J. D. Bude, "Soft breakdown in ultra-thin oxides," in Ultrathin $\mathrm{SiO}_{2}$ and High-K Materials for ULSI Gate Dielectrics, Materials Research Society Symposium Proceedings, H. R. Huff, C. A. Richter, M. L. Green, G. Lucovsky, and T. Hattori, Eds. Warrendale, PA: Materials Research Soc., 1999, vol. 567, pp. 301-306.

[183] C. Leroux, D. Blachier, O. Briere, and G. Reimbold, "Light emission microscopy for thin oxide reliability analysis," Microelectron. Eng., vol. 36, pp. 297-300, 1997.

[184] K. Okada and S. Kawasaki, "New dielectric breakdown model of local wearout in ultra thin silicon dioxides," in Extended Abstracts of the 1995 Int. Conf. on Solid State Devices and Materials, 1995, pp. 473-475.

[185] E. Miranda, J. Suñé, R. Rodríguez, M. Nafría, and X. Aymerich, "Detection and fitting of the soft breakdown failure mode in MOS structures," Solid-State Electron., vol. 43, pp. 1801-1805, 1999.

[186] _ _ "A function-fit model for the soft breakdown failure mode," IEEE Electron Device Lett., vol. 20, pp. 265-267, 1999.

[187] K. Okada and K. Taniguchi, "Electrical stress-induced variable range hopping conduction in ultrathin silicon dioxides," Appl. Phys. Lett., vol. 70, pp. 351-353, 1997.

[188] S. Takagi, M. Takayanagi, and A. Toriumi, "Experimental examination of physical model for direct tunneling current in unstressed/stressed ultrathin gate oxides," in Dig. 1999 Int. Electron Devices Meeting, 1999, pp. 461-464.
[189] Y. D. He, H. Guan, M. F. Li, B. J. Cho, and Z. Dong, "Conduction mechanism under quasibreakdown of ultrathin gate oxide," Appl. Phys. Lett., vol. 75, pp. 2432-2434, 1999.

[190] H. Guan, M. F. Li, Y. He, B. J. Cho, and Z. Dong, "A thorough study of quasibreakdown phenomenon of thin gate oxide in dual-gate CMOSFETs," IEEE Trans. Electron Devices, vol. 47, pp. 1608-1615, 2000.

[191] F. Crupi, R. Degraeve, G. Groeseneken, T. Nigam, and H. Maes, "On the properties of the gate and substrate current after soft breakdown in ultrathin oxide layers," IEEE Trans. Electron Devices, vol. 45, pp. 2329-2334, 1998.

[192] _ - "Investigation and comparison of the noise in the gate and substrate current after soft-breakdown," Jpn. J. Appl. Phys., vol. 38, pp. 2219-2222, 1999

[193] M. Depas, T. Nigam, and M. Heyns, "Definition of dielectric breakdown for ultra thin $(<2 \mathrm{~nm})$ gate oxides," Solid-State Electron., vol. 41, pp. 725-728, 1997.

[194] T. Pompl, H. Wurzer, M. Kerber, R. C. W. Wilkins, and I. Eisele, "Influence of soft breakdown on NMOSFET device characteristics," in Proc. 1999 Int. Reliability Physics Symp., 1999, pp. 82-87.

[195] W. K. Henson, N. Yang, and J. J. Wortmann, "Observation of oxide breakdown and its effects on the characteristics of ultra-thin-oxide nMOSFETs," IEEE Electron Device Lett., vol. 20, pp. 605-607, 1999.

[196] B. E. Weir, M. A. Alam, J. D. Bude, P. J. Silverman, A. Ghetti, F. Baumann, P. Diodato, D. Monroe, T. Sorsch, G. L. Timp, Y. Ma, M. M. Brown, A. Hamad, D. Hwang, and P. Mason, "Gate oxide reliability projection to the sub-2 nm regime," Semicond. Sci. Technol., vol. 15, pp. 455-461, 2000.

[197] B. Kaczer, R. Degraeve, G. Groeseneken, M. Rasras, S. Kubicek, E. Vandamme, and G. Badenes, "Impact of MOSFET oxide breakdown on digital circuit operation and reliability," in Dig. 2000 Int. Electron Devices Meeting, 2000, pp. 553-556.

[198] S. Lombardo, F. Crupi, and J. H. Stathis, "Softening of breakdown in ultra-thin gate oxide nMOSFETs at low inversion layer density," in Proc. 2001 Inst. Reliability Physics Symp., 2001, pp. 163-167.

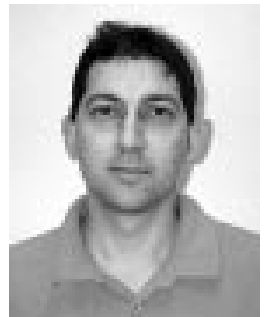

James H. Stathis received the B.S. degree in physics (summa cum laude) from Washington University, St. Louis, MO, in 1980, and the Ph.D. degree in physics from the Massachusetts Institute of Technology, Cambridge, in 1986.

In 1986, he joined the IBM Research Division The focus of his work at IBM has been the electrical properties of point defects in $\mathrm{SiO}_{2}$ and other insulators, including basic studies of defect structure using magnetic resonance and electrical measurement techniques, and the role of defects in wearout an 